Apidologie, 1974, 5 (4), 319-355.

\title{
MISE EN ÉVIDENCE D'UN CYCLE SAISONNIER DE LA TENEUR EN ACIDES CÉTO-9 ET HYDROXY-9 DÉCÊNE-2 OIIQUE DES TÊTES DE REINES VIERGES D'ABEILLE
}

\author{
Nachweis eines jahreszeitlichen Zyklus des Gehalts \\ an 9-Keto-2-decensäure und 9-Hydroxy-2-decensäure \\ in den Köpfen junger unbegatteter Königinnen \\ (Apis mellifica ligustica S.)
}

\author{
Janine PAIN, B. ROGER et J. THEURKAUFF \\ Station de recherches sur l'A beille et les insectes sociaux, I.N.R.A. \\ 91440 Bures-sur-Yvette
}

\begin{abstract}
SUMMARY
DETERMINATION OF A SEASONAL CYCLE FOR THE CONTENT IN 9-OXODEC AND 9-HYDROXYDEC-2-ENOIC ACIDS IN THE VIRGIN QUEEN'S HEADS OF BEES (Apis mellifera ligustica $\mathrm{S}$.)
\end{abstract}

During a period extending from March 1971 to March 1972, a queenrearing was achieved each month by means of a simple grafting or larvae. All the young larvae were issued from the same colony of Italian bees (A. m. ligustica S.). The rearing took place in two to four rearing colonies used in sucession. Two days before the queen eclosion, the queen cells were taken out and placed into an incubator, together with some forty bees nearly born, and about ten hive-bees which were fed with candy and pollen, the latter being stored before use at a temperature of $-25^{\circ} \mathrm{C}$.

On the 23 rd day of their eage-life, the virgin queens vers killed and weighed, the head being weighed separately. Then, the amount of 9-oxodec-2-enoic acid on the one hand and of 9-hydroxydec-2-enoic acid on the other hand was determined through gas chromatography. During the experiment, which covered 13 rearing series, 199 virgin queens were tested. The results were submitted to a statistical analysis.

The quantitative analysis revealed the existence of an annual cycle in the two investigated acids and a cycle in the weight of queens. The cycle of the 9-oxodec-2-enoic acid is the clearer and the more stable of the two. It is not dependent on the rearing colony. A first maximum in the production appears in June and a second, of lesser importance, in December. The 
lowest quantities are obtained in August and September, as well as in March. The cycle of the 9-hydroxydec-2-enoic acid is partially similar to that of the 9-oxodec-2-enoic acid but is delayed as compared to it. Concerning the weight of queens, a maximum was observed in May, one month before that of the 9-oxodec-2-enoic acid. The weights decrease regularly until November and there is no increase before spring.

A parallelism was etablished between the annual cycle of the 9-oxodec-2-enoic acid and that of the 10-hydroxydec-2-enoic acid produced by the worker bees which was detected previously (PAIN and Roger, 1970).

The relations between the secretion cycles of the pheromonal substances and different environmental factors (photoperiod, temperature, food resources) are discussed. The part played by the worker bees under this respect is also discussed, the worker bees being studied under variable physiological conditions according to which moment of the year they were selected to act as companions for the queens.

\section{RÉSUMÉ}

Au cours d'une période allant de mars 1971 à mars 1972 on a réalisé chaque mois dans un rucher couvert un élevage de reines en utilisant la technique du simple transfert de larves. Les jeunes larves provenaient toutes d'une même colonie d'abeilles italiennes $(A$. $m$. ligustica S.). Les élevages étaient faits dans deux à quatre colonies éleveuses servant alternativement. Deux jours avant l'éclosion des reines, les cellules royales étaient prélevées et mises à éclore en étuve en présence d'une cinquantaine d'abeilles naissantes recevant une alimentation à base de candi et de pollen, ce dernier conservé avant emploi à une température de $-25^{\circ} \mathrm{C}$.

$\mathrm{Au} 23^{\mathrm{e}}$ jour de leur vie en cagette les reines vierges étaient tuées et pesées, la tête faisant l'objet d'une pesée séparée. On dosait ensuite dans chaque reine, d'une part l'acide céto-9 décène 2 oïque, d'autre part l'acide hydroxy-9 décène-2 oïque, par chromatographie en phase gazeuse. Au cours de l'expérience qui a comporté 13 séries d'élevages, on a traité en tout 199 reines vierges. Les résultats ont fait l'objet d'une analyse statistique.

Les dosages ont montré qu'il existe un cycle annuel de chacun des deux acides étudiês et un cycle du poids des reines. Le cycle de l'acide céto-9 décène-2 oïque est le plus net et le plus stable. Il ne dépend pas de la colonie éleveuse. Un premier maximum de production apparaît en juin, un second, d'importance moindre, est atteint en décembre. Les quantités les plus faibles sont produites en août et septembre ainsi qu'en mars. Le cycle de l'acide hydroxy-9 décène-2 oïque se calque en partie sur celui de l'acide céto-9 mais avec un décalage dans le temps. En ce qui concerne le poids des reines on constate un maximum en mai, un mois avant celui de l'acide céto-9. Les poids diminuent régulièrement jusqu'en novembre et il n'y a pas de reprise avant le printemps.

On constate l'existence d'un parallélisme entre le cycle annuel de l'acide céto-9 décène-2 oïque et celui de l'acide hydroxy-10 décène-2 oïque produit par les ouvrières et précédemment mis en évidence (Pain et Roger, 1970).

On discute les rapports existant entre les cycles de secrétion des substances phéromonales et différents facteurs de l'environnement : photopériode, température, ressources alimentaires. On discute également du rôle joué par les ouvrières dans ce contexte ces ouvrières étant ellesmêmes dans des conditions physiologiques variables selon le moment de l'année où on les a prélevées pour servir d'accompagnatrices aux reines.

Descripteurs : Saison écologique, acide céto-9 décène-2 oïque, acide hydroxy-9 décène-2 oüque, acide hydroxy-10 décène-2 oüque, phéromone de déclenchement, phéromone d'amorçage, Apis m. ligustica, reine insecte, poids.

\section{INTROD UCTION}

L'acide céto-9 décène-2 trans oïque est un acide cétonique insaturé présent dans les glandes mandibulaires des reines d'abeilles. Il a été obtenu à l'état 
pur à partir d'extraits alcooliques de reines entières, d'extraits éthérés de têtes et de glandes mandibulaires de reines vierges ou fécondes d'Apis mellifica (Barbier et al. 1960 - Butler, Callow, Johnston, 1961 - Callow, Johnston, 1960 - GARY, 1962).

Chez un groupe de reines vierges d'âge connu, nous avons trouvé une quantité moyenne de $108,2 \mu$ g (PAIN, Barbier, Roger, 1967). Les quantités moyennes de phéromone dosable trouvées par d'autres auteurs semblent être du même ordre de grandeur : $130 \mu \mathrm{g} \perp 15 \%$ dans les têtes de reines de 5 à 10 jours, $150 \mu \mathrm{g} \pm 15 \%$ dans les têtes de reines fécondes de quelques semaines (Butler, Paton, 1962), $200 \mu \mathrm{g}$ dans un extrait de têtes de reines (Callow, Chapman, Paton, 1964). Ces résultats sont en accord avec ceux plus récents de Shearer et al. (1970).

Les mêmes auteurs se sont efforcés de le mettre en évidence dans les reines des 3 autres espèces que comporte le genre Apis. C'est ainsi que dans des solutions éthyliques de conservation de reines fécondes d'Apis cerana et $A$. florea, Butler et ses collaborateurs (1967) ont signalé la présence d'une substance dont le temps de rétention est identique à celui de l'ester méthylé de l'acide céto-9 décène- 2 oïque. Les auteurs concluent à la présence de cet acide chez les deux espèces. Les quantités sont évaluées approximativement à $10 \mu \mathrm{g}$ au maximum d'acide par reine.

Chez Apis dorsata, une estimation quantitative à partir d'extraits céphaliques donne les valeurs de 100 à $200 \mu \mathrm{g}$ d'acide céto-9 décène-2 oïque. Ces quantités sont du même ordre de grandeur que celles mises en évidence chez Apis cerana et $A$. mellifera par Shearer et al, 1970. Ces derniers résultats ont été confirmés par Sannasi et Sundara Rajulu (1971).

La recherche de cet acide dans les reines d'espèces différentes semblait retenir tout l'intérêt des chercheurs. Par contre, le facteur « saison » n'a jamais été pris en considération. Or, dès le début de nos recherches, nous avions pensé que la phéromone royale n'est pas émise de façon continue. Pour étudier sa production, nous avions mesuré pendant plusieurs mois, le pouvoir d'attraction de reines vivantes, ce pouvoir étant lié à la présence de l'acide céto-9 décène-2 oïque (Barbier, Pain, 1960). Les reines étudiées n'avaient pas pu être suivies pendant toute une année. Nous avions cependant entrevu un processus de sécrétion qui semblait cyclique, les reines ne présentant pas le même pouvoir d'attraction envers les ouvrières, selon le mois où l'observation était faite.

Par la suite et pour un autre acide gras, l'acide hydroxy-10 décène-2 oïque présent dans les sécrétions céphaliques des ouvrières, nous avons mis en évidence l'existence d'une variation annuelle (PAIN, Roger, 1970).

D'après les travaux de Brown et Felauer, 1961, il est possible d'envisager une parenté biochimique entre ces deux acides. 
Il nous a donc paru intéressant de rechercher si la production de l'acide céto-9 décène- 2 oïque présente elle aussi une variation annuelle du même type.

Parallèlement, nous avons pensé qu'il serait utile de doser dans les extraits royaux l'acide hydroxy-9 décène-2 oïque. La synthèse en a été réalisée par différents auteurs (Barbier, Hügel, 1961 - Brown, Felauer, Smith, 1962 Eiter, 1962). On l'a signalé dans les extraits de têtes de reines, mais en plus petites quantités que l'acide céto-9 décène-2 oïque (Callow, Chapman, Paton, 1964). Il est également présent, en faible proportion, dans le mélange d'acides carboxyliques isolés de la gelée royale (Brown, Felaver, 1961). Son rôle est jusqu'ici moins connu que celui de l'acide céto-9 décène-2 oïque.

L'acide hydroxy-9 décène-2 oïque est une phéromone sexuelle moins attractive que l'acide céto-9 décène-2 oïque (Butler, Fairey, 1964). C'est une phéromone stabilisatrice des ouvrières essaimantes (Butler, Callow, Chapman, 1964) et inhibitrice de la construction des cellules royales (ButLer, CALlow, 1968) en association avec l'acide céto-9 décène-2 trans oïque. Ce serait un intermédiaire entre l'acide céto-9 décène-2 oïque et l'acide hydroxy-10 décène-2 oïque. Ce dernier acide serait lui-même un précurseur de l'acide céto-9 décène-2 oïque (Brown, Felauer, 1961. Dans certaines conditions expérimentales, de petites quantités d'acide hydroxy-9 décène-2 oïque sont présentes dans le tube digestif des ouvrières qui ont ingéré de l'acide céto-9 décène-2 oïque marqué (Johnston, LAw, WEAvER, 1965).

L'évaluation des quantités enregistrées sur les chromatogrammes en même temps que l'acide céto-9 décène- 2 oïque est susceptible de nous renseigner sur son rythme propre de formation chez les reines.

L'existence d'un cycle saisonnier de la production des deux acides ne paraît pas avoir fait jusqu'ici l'objet de recherches.

Puisque nous disposions d'assez grandes quantités de matériel biologique, nous avons songé à effectuer des pesées de têtes et des pesées de corps entiers de reines, conservés à l'état frais dans un congélateur en vue des dosages.

Nous ne pensons pas, a priori, qu'il existe un rapport entre les sécrétions phéromonales et le poids des reines. Il nous a paru cependant intéressant d'utiliser toutes les données que nous avions obtenues au cours de ce travail pour rechercher une éventuelle corrélation.

D'autre part, la variation saisonnière du poids des reines n'ayant fait l'objet jusqu'ici que de rares travaux (Skrobal, 1958 - Shimanova, 1966 Mirza, Duduman, Dragan, 1967 - Selemenev, 1970), nous avons utilisé les données disponibles pour l'étudier.

D'après les auteurs russes (Shimanova, Selemenev), il semblerait que le poids des reines d'Apis mellifica caucasica soit plus élevé au mois de juin (Selemenev, 1970). Pour Skrobal (1958) les expériences poursuivies en Tchécoslovaquie indiquent également que le poids des reines augmente au 
cours du printemps et de l'été, pour diminuer ensuite. Pour les auteurs roumains (Mirza et al, 1967) le poids des reines à l'éclosion chez Apis mellifica carnica augmenterait du mois de juin au mois d'août. Nous rappelons au sujet de ce travail que K. Weiss (1967) a signalé que le poids des reines à l'éclosion ne lui paraissait pas se prêter à des comparaisons précises en raison des différences individuelles importantes qui apparaissent à ce stade. Hoopingarner et Farrar (1959) considèrent que la pesée des nymphes est la méthode la plus sûre. Le poids moyen des reines adultes peut toutefois être pris en considération à condition de prendre quelques précautions.

Avant d'aborder les expériences proprement dites, nous voudrions rappeler les rôles divers et importants que joue l'acide céto-9 décène-2 oïque dans la colonie. C'est :

$1^{0}$ une phéromone attractive sexuelle,

$2^{\circ}$ une phéromone attractive d'agrégation envers les ouvrières essaimantes,

$3^{0}$ une phéromone d'interattraction des ouvrières, dans certaines conditions,

$4^{\circ}$ une phéromone inhibitrice de la construction des cellules royales,

$5^{\circ}$ une phéromone inhibitrice du développement des ovaires des ouvrières (Butler, 1967 - Pain, 1973).

Sa présence conditionne donc en partie la vie de la colonie en agissant sur le comportement et la physiologie des ouvrières. Son action multiple justifie l'étude approfondie que nous avons entreprise. Il est probable, et c'est l'objet de ce travail, que sa production au cours d'une année n'est pas régulière. Elle doit suivre un certain rythme en rapport avec des phénomènes biologiques à caractère cyclique qui règlent la vie de la ruche.

\section{MATÉRIEL ET MÉTHODES}

\section{Le rucher couvert}

L'élevage de reines a été mené pendant un an dans un rucher couvert de la Station de Recherches sur l'Abeille et les Insectes Sociaux à Bures-sur-Yvette. Il s'agit d'un bâtiment de bois, bien calorifugé, dans lequel on peut installer 15 ruches par groupe de 5 . Les ruches sont mises en place dès le début de septembre de chaque année.

Le rucher est chauffé d'octobre à avril. La température est règlée à $20^{\circ} \mathrm{C} \pm 1^{\circ}$. Pendant cette même période, les ruches reçoivent régulièrement du pollen présenté dans des mangeoires de $300 \times 100 \times 13 \mathrm{~mm}$ que l'on introduit à la partie inférieure du corps de ruche spécialement aménagée pour les recevoir. Nous utilisons un pollen en pelotes de l'année, non séché et conservé à $-25{ }^{\circ} \mathrm{C}$. Il est additionné d'un peu d'eau et de miel pour le transformer en pâte. Celle-ci contient pour $3 \mathrm{~kg}$ de pollen frais $700 \mathrm{~g}$ de miel et $700 \mathrm{ml}$ d'eau. On laisse macérer quelques heures. Chaque mangeoire peut contenir $600 \mathrm{~g}$ de pâte.

Une surveillance régulière des réserves de pollen est nécessaire pour éviter un ralentissement ou un arrêt de ponte de la reine. La méthode utilisée permet un contrôle facile de la 
consommation de la nourriture, sans perturber la colonie. Les ruches qui consomment peu ou mal le pollen sont toujours celles dans lesquelles on ne trouve pas ou presque pas de couvain.

Chaque jour, les ruches reçoivent $100 \mathrm{ml}$ de sirop de sucre à $50 \%$ tiédi et chaque semaine un complément de $120 \mathrm{ml}$ d'eau ordinaire.

Le rucher couvert nous permet tout au long de l'année d'obtenir les jeunes larves nécessaires à l'élevage de reines. Il nous fournit en même temps les ouvrières naissantes dont nous avons besoin pour accompagner les reines produites.

\section{L'élevage de reines}

Au rucher couvert, tous les mois pendant un an, du mois de mars 1971 au mois de mars 1972, nous avons fait des séries d'élevages de reines selon la technique de VuiLlaume (1958) modifiée par l'un de nous.

De jeunes larves indifférenciées sont prélevées dans des cellules d'ouvrières pour être déposées à l'intérieur de cupules en cire de la dimension d'une cellule royale. Avant le transfert des larves, les cupules sont “familiarisées " par un séjour de 8 à 12 heures dans une ruche normale. Nous avons effectué un transfert simple : la larve déposée n'est pas remplacée le $2^{\mathrm{e}}$ jour comme on le fait dans la technique de double " greffage ».

Pour une même série d'élevages, les très jeunes larves proviennent toujours de la même colonie d'abeilles italiennes. Cependant, au cours de l'année, il nous est arrivé par deux fois d'être obligés de les prélever dans une autre ruche (séries 3 et 9 ). Pour deux autres séries $\left(n^{\circ} 10\right.$, décembre 1971 - no 14 , février 1972) les larves proviennent de deux colonies donneuses différentes mais de même race.

Les larves transférées sont nourries par une colonie éleveuse. Celle-ci est une ruche normale au-dessus de laquelle on a placé une grille à reine surmontée d'un deuxièr.ie corps de ruche. L'élevage royal s'effectue dans la partie supérieure qui est orpheline lorsqua la colonie est très peuplée. Lorsqu'elle possède une population plus réduite, la ruche est séparée en deux par une cloison verticale. En hiver, la technique d'orphelinage de la ruche éleveuse a été adaptée aux nécessités. On ne divise plus la ruche par une grille à reine : c'est la colonie entière qui est orphelinée (séries 9 à 13).

Pendant toute la durée de l'expérience, nous avons utilisé quatre ruches éleveuses. Elles étaient de race italienne pure et possédaient des reines sceurs âgées d'un an en 1971. Par mesure de sécurité, dans chaque série, on a toujours utilisé deux ruches éleveuses pour être sûr d'obtenir des reines. Nous n'avons prélevé en vue des dosages que les reines provenant de la ruche éleveuse qui avait donné le plus grand nombre de cellules royales. Nous avons procédé ainsi jusqu’à la dernière expérience (série 13). Chaque ruche éleveuse recevait 23 larves à nourrir. A la série suivante, les deux groupes de larves étaient confiés aux deux autres ruches. Nous avons opéré de cette façon afin que les quatre ruches utilisées pendant un an, n'assurent un élevage de reines que tous les deux mois environ.

Les cellules royales operculées étaient prélevées dans la ruche éleveuse 48 heures avant la naissance des reines. Elles étaient introduites isolément dans des cagettes expérimentales Pain, 1966). Dans celles-ci, on mettait en même temps une quinzaine d'ouvrières d'âge indéterminé prises sur le cadre d'élevage. Un ou deux jours après la naissance des reines, on introduisait 40 ouvrières de un à trois jours. Elles recevaient du pollen, du candi et de l'eau (Roger, 1971).

Toutes les reines restaient à l'étuve à $32 \pm 1{ }^{\circ} \mathrm{C}$. La plupart d'entre elles (11 à 17 sauf dans la série $8: 6$ reines) étaient tuées à l'âge de $23 \pm 1$ jours. Après une anesthésie très rapide au gaz carbonique, elles étaient conservées dans un congélateur à $-25^{\circ} \mathrm{C}$, dans des tubes individuels. Elles y restaient jusqu'au moment du dosage des acides. Les autres reines étaient utilisées dans d'autres buts expérimentaux.

\section{Dosage des acides céto-9 et hydroxy-9 décène-2 oüque}

La technique de dosage par chromatographie en phase gazeuse de l'acide céto-9 décène-2 oĩque a déjà été décrite (PAIN, BARBIER, Roger, 1967). Elle est identique à celle que nous avons utilisée pour doser l'acide hydroxy-10 décène-2 oíque dans les têtes des ouvrières (PAIN, Roger, 1970). Pour doser l'acide hydroxy-9 décène-2 oïque les conditions de travail sont les mêmes.

Les extraits sont obtenus à partir de broyats de têtes de reines dans l'éther. Ils sont amenés à sec puis méthylés pour être repris par des quantités appropriées d'hexane. Les 


\begin{tabular}{|c|c|c|c|c|c|c|c|c|c|c|c|c|c|c|}
\hline 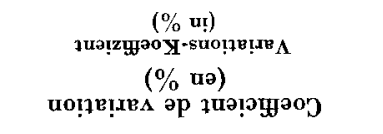 & $\begin{array}{l}a \\
\tilde{b}\end{array}$ & $\frac{\infty}{15}$ & $\begin{array}{l}\mathcal{a} \\
\stackrel{+}{*}\end{array}$ & | & & is & धे & 畓 & $\begin{array}{l}\text { ýd } \\
\text { in }\end{array}$ & & $\dot{0}$ & 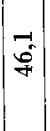 & \begin{tabular}{c}
\multirow{1}{*}{} \\
$\infty$ \\
$\infty$
\end{tabular} & \\
\hline 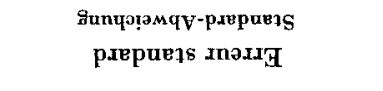 & 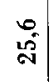 & $\stackrel{0}{0}$ & $\stackrel{\oplus}{=}$ & 交 & & 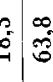 & is & $\sigma^{\circ}$ & 文 & సิ & S. & $\stackrel{\sim}{\sim}$ & $\stackrel{*}{*}$ & \\
\hline 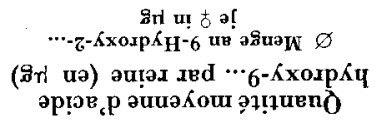 & 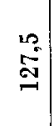 & $\vec{m}$ & $\stackrel{0}{\circ}$ & 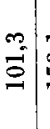 & & & คิं & $\begin{array}{l}0 \\
\dot{f} \\
\end{array}$ & $\mid \begin{array}{l}4 \\
0 \\
05\end{array}$ & $\hat{\sigma}$ & : & $\mid \begin{array}{c}\infty \\
\text { î̀ } \\
\end{array}$ & बे & \\
\hline 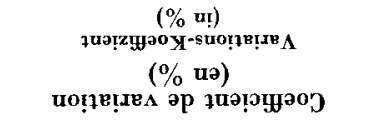 & $\begin{array}{l}0 \\
5 \\
5\end{array}$ & $\begin{array}{l}m \\
\text { in } \\
\text { in }\end{array}$ & 字 & $\frac{N}{m}$ & $\begin{array}{c}7 \\
0 \\
0\end{array}$ & & 豙 & 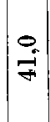 & ลิ & $\left(\begin{array}{c}0 \\
8\end{array}\right.$ & 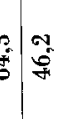 & is & $\mid \begin{array}{c}0 \\
\infty \\
\infty\end{array}$ & \\
\hline 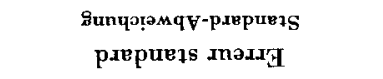 & న్ & $\left|\begin{array}{c}1 \\
\infty \\
\infty \\
\cdots\end{array}\right|$ & $\mid$ & \begin{tabular}{l}
$\infty$ \\
$\dot{\delta}$ \\
\hdashline
\end{tabular} & : & 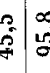 & is & Lิ & $\left|\begin{array}{c}\infty \\
\dot{m} \\
\dot{m}\end{array}\right|$ & 毒 & Din & 兽 & 我 & \\
\hline 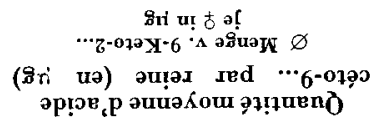 & 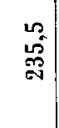 & $\stackrel{\Xi}{\Xi}$ & $\mid$ & 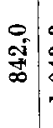 & 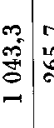 & : & ฟึ & 昰 & 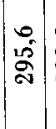 & 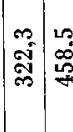 & | & $\mid$ & $\stackrel{N}{2}$ & \\
\hline 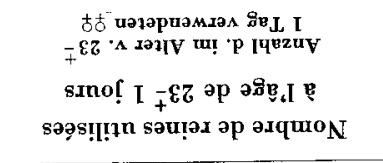 & $\Xi$ & $\mathbf{\Xi}$ & $\Xi$ & $\simeq$ & $\mathfrak{9}$ & $\stackrel{9}{\rightarrow}=$ & 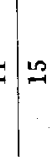 & 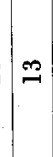 & 0 & $\stackrel{\sim}{\sim}$ & \pm & $\stackrel{20}{9}$ & 10 & 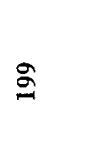 \\
\hline 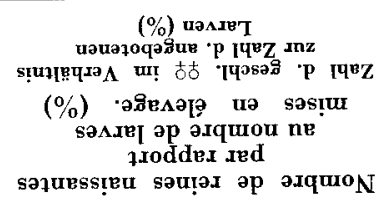 & \&ें & $\mid \begin{array}{c}0 \\
0 \\
0 \\
0\end{array}$ & $\frac{m}{a}$ & 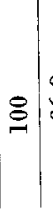 & 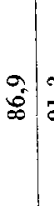 & 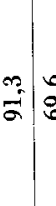 & $\stackrel{n}{\circ}$ & $\mid \begin{array}{l}0 \\
i \\
\infty\end{array}$ & $\vec{s}$ & \begin{tabular}{l|l}
0 & 0 \\
if & $\infty$ \\
in & $\infty$
\end{tabular} & : & $\mid \begin{array}{l}0 \\
0 \\
0 \\
0\end{array}$ & o̊ & \\
\hline 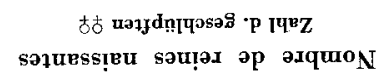 & নి & $\infty$ & $\vec{\sim}$ & กี & సิ & $\overrightarrow{\mathrm{N}}$ & $\bar{a}$ & 19 & 10 & $\stackrel{\sim}{\sim}$ & 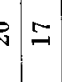 & 12 & 10 & ్ֶ \\
\hline 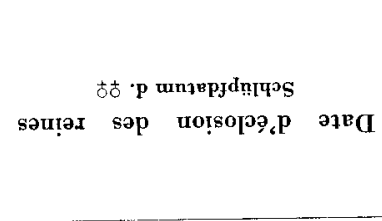 & $\begin{array}{l}\overrightarrow{0} \\
\dot{\tilde{\theta}} \\
\dot{\phi} \\
\dot{0}\end{array}$ & $\left|\begin{array}{c}F \\
\vec{d} \\
\dot{\vec{i}} \\
\dot{0} \\
\dot{g}\end{array}\right|$ & & 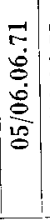 & 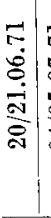 & 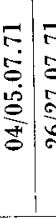 & 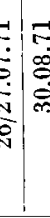 & $\frac{\pi}{5}$ & 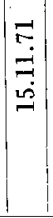 & 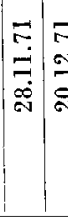 & 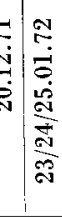 & 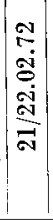 & 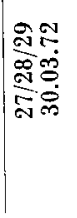 & 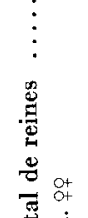 \\
\hline 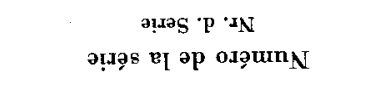 & - & No & $\infty$ & + & $\approx$ & $n 2$ & $i 0$ & 10 & $\infty$ & 10 & $\Xi=$ & $\boldsymbol{I}$ & $m$ & 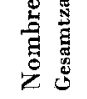 \\
\hline
\end{tabular}


TaBl. 1 bis. - Récapitulation : Poids des reines des séries 1 à 13 (1) de mars 1971 à mars 1972

Tав. I f. - Wiederholung : Königinnengewichted. Serien I-13 (1) v. März 1971 bis März 1972

\begin{tabular}{|c|c|c|c|c|c|c|}
\hline 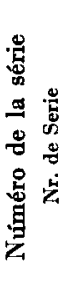 & 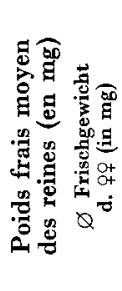 & 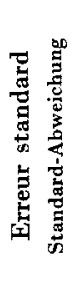 & 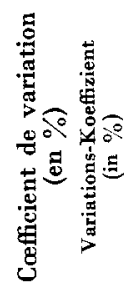 & 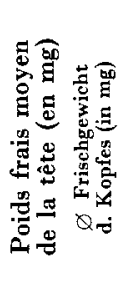 & 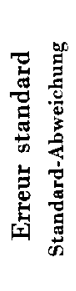 & 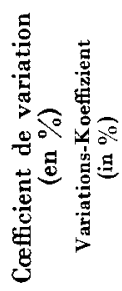 \\
\hline 1 & 133,7 & 2,7 & 7,6 & 10,3 & 0,13 & 5,0 \\
\hline 2 & 148,8 & 3,8 & 9,0 & 11,0 & 0,26 & 8,0 \\
\hline 3 & 180,9 & 2,6 & 5,4 & 11,8 & 0,19 & 5,6 \\
\hline 4 & 170,3 & 2,4 & 5,9 & 12,2 & 0,09 & 3,4 \\
\hline $4^{\prime}$ & 164,1 & 2,7 & 5,7 & 11,5 & 0,11 & 3,4 \\
\hline 5 & 171,8 & 2,3 & 5,2 & 11,9 & 0,13 & 4,1 \\
\hline $5^{\prime}$ & 165,7 & 4,2 & 8,5 & 11,3 & 0,12 & 3,9 \\
\hline 6 & 159,8 & 3,1 & 7,6 & 11,0 & 0,18 & 6,9 \\
\hline 7 & 161,4 & 4,5 & 10,0 & 11,0 & 0,19 & 6,2 \\
\hline 8 & 170,9 & 5,2 & 7,5 & 11,3 & 0,29 & 6,4 \\
\hline 9 & 148,9 & 3,8 & 8,9 & 10,4 & 0,20 & 6,5 \\
\hline 10 & 162,4 & 0,9 & 2,2 & 11,0 & 0,35 & 11.7 \\
\hline 11 & 152,1 & 4,4 & 10,5 & 11,1 & 0,19 & 6,8 \\
\hline 12 & 144,3 & 3,8 & 10,2 & 10,6 & 0,21 & 8,0 \\
\hline 13 & 143,8 & 3,0 & 8,4 & 9,8 & 0,20 & 8,3 \\
\hline
\end{tabular}


quantités de substances de synthèse servant de référence pour chaque série de dosage ont été au minimum de $1 \mathrm{mg}$. On en prépare une solution dans 100 à $200 \mu \mathrm{l}$ d'hexane. Nous avons injecté de 1 à $3 \mu l$ de solution de référence (acides céto-9 et hydroxy-9 décène-2 oïque des synthèse) et de 1 à $5 \mu l$ d'extraits céphaliques de reines, la quantité utilisée variant en fonction de la teneur en acides.

L'acide céto-9 décène-2 oïque se présente sous forme de cristaux, l'acide hydroxy-9 décène-2 oïque à l'aspect d'une huile. Il est de ce fait plus difficilement quantifiable.

Nous avons utilisé un enregistreur W.G. Pye and Co. Ltd., Cambridge. G. B., et un chromatographe gaz liquide (chromagas Profit, C.G. 10, licence C.N.R.S.) à détection par ionisation de flamme. Il comporte une colonne spiralée de 2 mètres. Son diamètre est de $1 / 4$ de pouce. La colonne est remplie de Kiselgur 80/100 mesh imprégné de P.E.G. à $15 \%$.

Le temps de rétention de l'ester de l'acide céto-9 décène-2 oïque est de 8 minutes, celui de l'ester de l'acide hydroxy-9 décène-2 oïque de dix minutes. Les limites de détection sont de l'ordre de $0,01 \mu \mathrm{g}$. Nous avons effectué deux injections par extrait de reine. La substance témoin de synthèse est injectée après 4 injections d'extraits céphaliques, soit toutes les deux reines.

Les dosages des extraits céphaliques sont obtenus par comparaison des aires de pics d'enregistrement avec celles fournies par des échantillons d'esters méthyliques des acides céto- 9 et hydroxy-9 décène -2 oïque de synthèse.

Les variations maximales enregistrées au cours d'une même série de mesure sont de l'ordre de $10 \%$.

Pesée des reines

Les reines conservées séparément à l'état frais dans un congélateur à $-25{ }^{\circ} \mathrm{C}$ sont pesées sur une balance au $1 / 10$ de mg juste avant la préparation des têtes et après réchauffement à la température du laboratoire. La tête est pesée ensuite séparément.

\section{RÉSULTATS}

Les expériences ont débuté à la fin de février 1971. A partir de cette date, nous avons élevé une série de reines chaque mois très régulièrement. Au cours des mois de juin et de juillet, époque du plein développement des colonies, nous avons pu élever deux séries supplémentaires $\left(n^{0} 4^{\prime}\right.$ et $\left.n^{0} 5^{\prime}\right)$, soit au total 15 séries (Tableau 1). Sur les figures 1 (A) et (B) et 2 (A) et (B), les quantités moyennes d'acides et les poids ont été regroupés pour les 2 séries de juin (série 4 ) et pour les 2 séries de juillet (série 5).

Les courbes ont été établies en conséquence.

La dernière expérience de mars 1972 comporte 4 groupes de reines (séries 13 (1) (2) (3) (4) au lieu des deux groupes habituels.

Le nombre des reines obtenues à la naissance n'est pas toujours le même. Il varie en fonction de la période d'élevage. De mars à septembre 1971, nous obtenons en moyenne $86,9 \%$ de reines naissantes et d'octobre à mars 1972 , $65,2 \%$ seulement. Le mois de novembre est celui où nous avons la plus faible proportion de reines naissantes (26 et $52 \%$ ) (Tableau 1 et figure 3) (B).

On considère que l'expérimentation dans chaque série s'étend sur une période de $35 \pm 1$ jours. Pour établir la plupart des courbes, nous avons porté en abscisses les dates de naissance des reines.

Dans chaque série, les reines ont fait l'objet de dosages individuels. 

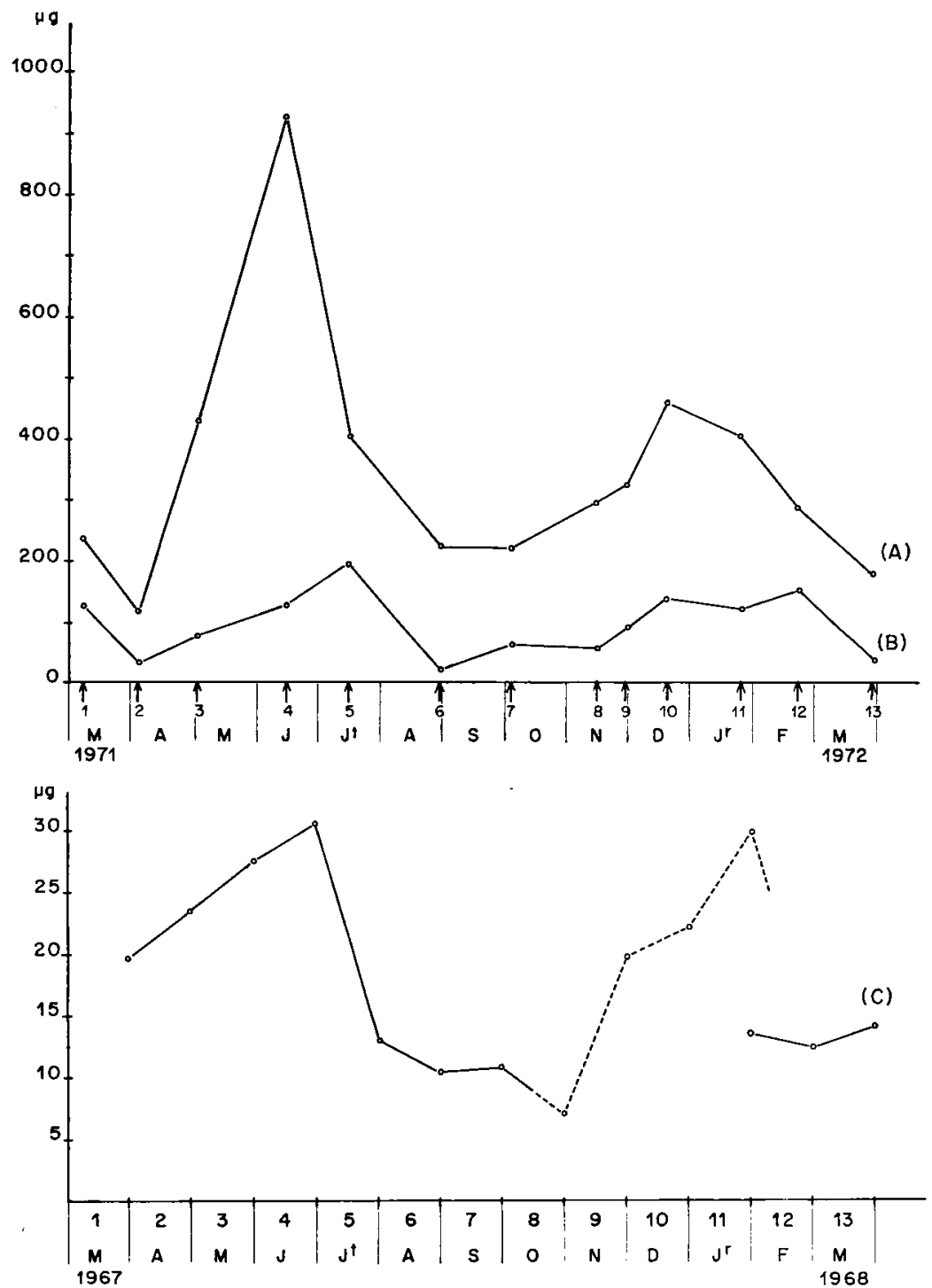

FIG. 1

(A) $=\mu g$ d'acide céto-9 décène-2 öque obtenus dans les têtes de reines vierges ( $23 \pm 1$ jours).

(B) $=\mu \mathrm{g} d^{\prime}$ 'acide hydroxy-9 décène-2 ö̈que obtenus dans les têtes de reines vierges ( $\overline{23} \pm 1$ jours).

(C) $=$ ug d'acide hydroxy-10 décène-2 oüque obtenus dans les têtes des ouvrières (Résultats de mars 1967 à mars 1968).

La courbe en trait plein se rapporte à des dosages dans les têtes d'ouvrières âgées de $16 \pm 2$ jours. La courbe en pointillé se rapporte à des dosages dans les têtes d'ouvrières d'âge croissant (séries 8 à 11), âgées successivement de 35 à 140 jours.

ABB. 1

(A) = $\mu$ g 9-Keto-2-decensäure aus den Köpfen unbegatteter $q \uparrow(23 \pm 1$ Tag alt $)$

(B) = $\mu g$ 9-Hydroxy-2-decensäure aux den Köpfen unbegatteter $9{ }^{-}(23 \pm 1$ Tag alt $)$

(C) $=\mu g$ 10-Hydroxy-2-decensäure aus Arbeiterinnenköpfen (Ergebnisse v. März 1967 bis März 1968)

Die ausgezogene Kurve bezieht sich auf die Bestimmungen d. Köpfe $16 \pm 2$ Tage alter Arbeiterinnen. Die punktierte Kurve bezieht sich auf die Bestimmungen d. Köpfe von Arbeiterinnen zunehmenden Alters (Serie 8 bis 11), zwischen 35 u. 140 Tage alt. 


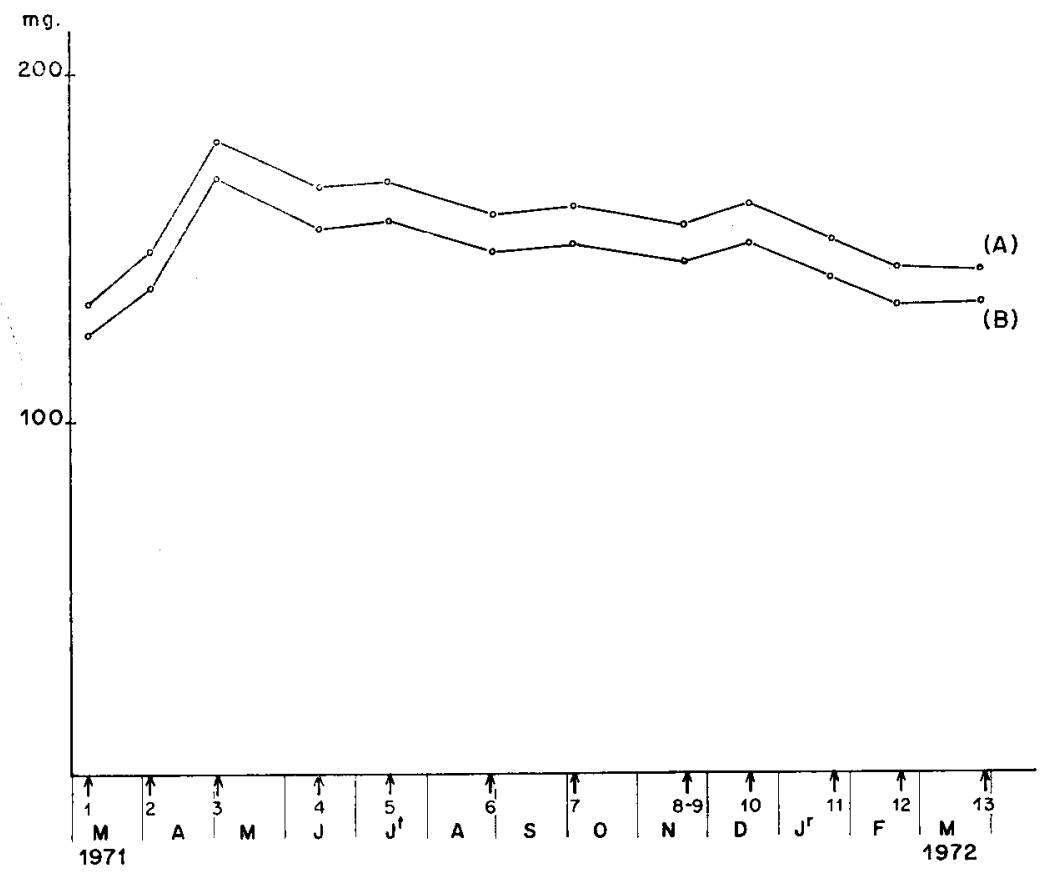

FIG. 2

(A) Poids frais moyen des reines entières (en $\mathrm{mg}$.)

(B) Poids frais moyen du thorax et de l'abdomen des reines (en mg.)

ABB. 2

(A) $=\varnothing$ Frischgewicht der ganzen 우 (in mg)

(B) $=\varnothing$ Frischgewicht von Thorax u. Abdomen d. ㅇ (in mg)

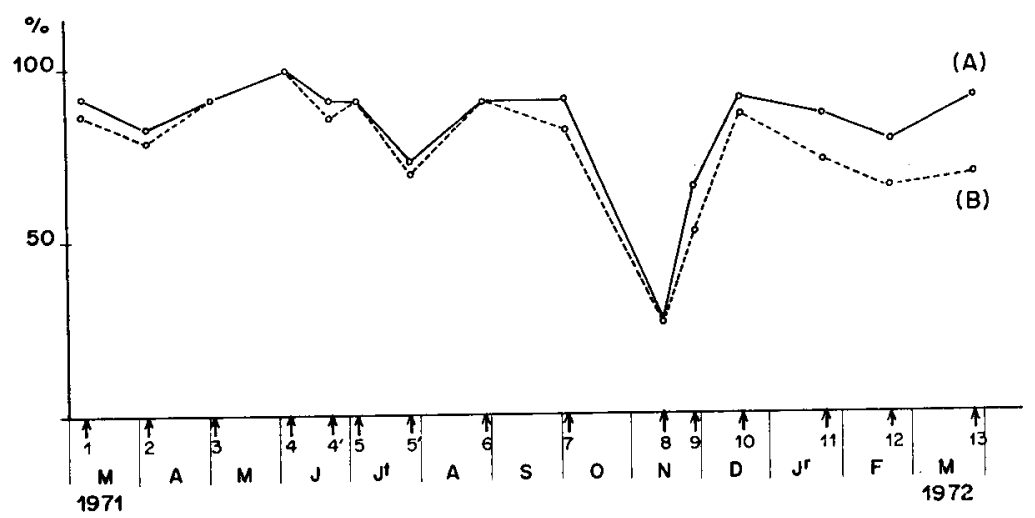

Frg. 3

(A) Pourcentage de larves acceptées.

(B) Pourcentage de reines naissantes.

AвB. 3

(A) $=\%$ d. angenommenen Larven

$(\mathrm{B})=\%$ d. geschlïpften $q \uparrow$ 


\section{1. - Dosage de l'acide céto-9 décène-2 ö̈que dans les têtes de reines âgées de $23 \pm 1$ jours}

Comme nous l'avons indiqué précédemment, nous n'avons conservé en vue des dosages qu'un seul groupe de reines. Elles proviennent toujours de la ruche éleveuse qui a donné le nombre le plus élevé de cellules royales. Sur la figure 1 (courbe A) sont portées les valeurs moyennes de chaque série d'élevage. Les valeurs correspondent aux quantités trouvées dans les têtes de reines âgées de 23 jours.

La courbe met en évidence une augmentation des valeurs moyennes de l'acide céto- 9 décène- 2 oïque à partir de la série 3 (reines écloses le 2 mai). La quantité moyenne d'acide est déjà élevée (426 $\mu \mathrm{g}$.$) . Elle représente environ$ le double de celles qu'on peut trouver dans la littérature.

Dans la série no 4 du début de juin (reines écloses le 5 et 6 juin), nous obtenons $842 \mu \mathrm{g}$. d'acide céto-9 décène-2 oïque. C'est la première fois que nous enregistrons de telles quantités de phéromone royale. Ces résultats nous ont incités à répéter l'expérience beaucoup plus tôt que prévu. Quinze jours après (série $n^{\circ} 4^{\prime}$ ) nous avons élevé un autre groupe de reines. Les quantités d'acide sont encore plus élevées ( $1043,4 \mu \mathrm{g}$.$) .$

Nous assistons depuis le début de mai à une augmentation très nette du taux de la substance royale. Cette augmentation confirme l'hypothèse de l'existence d'un cycle de production de l'acide céto-9 décène- 2 oïque en rapport avec la saison et le développement des colonies.

Dans la série no 5 du début de juillet (reines écloses les 4 et 5 juillet), les quantités d'acide sont nettement moins importantes. Afin de confirmer la baisse enregistrée par rapport aux résultats des séries précédentes (séries 4 et $4^{\prime}$ ) nous avons refait une autre série de reines (série $n^{\circ} 5^{\prime}$ ). Nous obtenons des quantités d'acide céto-9 décène- 2 oïque plus élevées que celles de la série 5 . Elles sont toutefois plus faibles que celles des séries de juin. Nous avons cherché à expliquer cette remontée sensible de la quantité de phéromone royale. Dans la série no 5 , sur 23 larves transférées : 21 reines sont nées (soit $91,3 \%$ ) alors que dans la série $5^{\prime}, 16$ reines seulement ont éclos(soit $69,6 \%$ ). Nous pensons que les larves acceptées dans la série 5' ont été mieux nourries. Il est possible qu'une meilleure alimentation des larves, à certaines périodes de l'année, soit susceptible d'avoir un effet sur la production ultérieure d'acide céto-9 décène-2 oïque.

Pour les deux séries de juin, on peut donner la même explication : 20 reines sont nées dans la série $4^{\prime}(86,9 \%)$ alors que dans la série 4,23 reines ont éclos $(100 \%)$. Or dans la série 4 , les quantités d'acides sont les plus fortes. 
Le nombre de larves de reines prises en élevage en été parait done avoir une certaine importance. Par contre, lorsque la colonie est au début ou à la fin de son développement, le nombre de reines obtenues à la naissance ne semble pas avoir la même répercussion sur la production d'acide céto-9 décène-2 oïque.

Quels que soient les mécanismes impliqués dans la production de la phéromone royale, on peut dire que les quantités d'acide céto-9 décène- 2 oïque augmentent depuis le début du mois de mai jusqu'à la fin du mois de juin. C'est en juin que se place le maximum. La quantité d'acide diminue ensuite plus ou moins rapidement dans le courant de juillet. Elle atteint les valeurs les plus basses à la fin de l'été (août, septembre). A partir d'octobre, nous avons eu des difficultés pour trouver des larves et du couvain dans les colonies du rucher couvert. C'est la raison pour laquelle les élevages de reines ont été effectués avec un léger retard soit vers la mi-novembre. Dans la série 8, nous n'avons obtenu que 6 reines. Ce nombre étant insuffisant, nous avons recommencé un élevage treize jours plus tard (série 9). Nous désirions par ailleurs vérifier la tendance à une nouvelle remontée des teneurs en acide céto-9 décène-9 oïque.

Effectivement, les quantités d'acide augmentent à nouveau à partir de la mi-novembre. Cette augmentation se poursuit jusqu'au mois de décembre. Nous obtenons un deuxième maximum mais moins important qu'en juin. A partir de janvier et jusqu'en mars 1972, nous assistons une nouvelle fois à une diminution progressive de la quantité de phéromone royale. La série 1 (mars 1971) et la série 13 (1) (mars 1972) indiquent les valeurs assez comparables qui oscillent autour de $200 \mu \mathrm{g}$. d'acide céto-9 décène-2 oïque.

Chaque tête de reine ayant fait l'objet d'un dosage individuel nous avons pu calculer pour chaque série, la variance. Elle nous a permis d'effectuer un test $\mathbf{t}$ de différence de moyennes entre les différents points mensuels de la courbe A de la Fig. 1 (Tableau 5). Les résultats indiquent des valeurs très significatives $(\mathrm{P}=1 \%$ oo $)$ entre les séries 1 et 2,2 et 3,3 et 4 . On peut done affirmer que les quantités d'acide céto-9 décène-2 ö̈que augmentent bien de mars à juin. En juin, les 2 séries 4 et 4 ' analysées séparément donnent des valeurs de $t$ qui ne sont pas significatives. Les teneurs en acides obtenues au début de juin et à la fin de ce mois sont les mêmes. Les quantités d'acide céto-9 décène-2 oïque diminuent ensuite d'une façon très significative $(P=1 \%)$ jusqu'à la fin d'août. Puis nous obtenons des valeurs non significatives entre les séries 6 et 7,7 et 8,8 et 9,9 et 10,10 et 11,11 et 12. On pourrait conclure que les quantités d'acide céto-9 décène-2 oïque n'augmentent pas en hiver. Cependant, nous avons des valeurs très significatives $(P=1 \%)$ entre les séries 6 ou 7 et 10 et entre les séries 10 et 13. Par conséquent, on admet qu'il y a en hiver, une augmentation des quantités d'acide céto-9 décène-2 oïque. 
Enfin, la comparaison entre les 2 séries 1 (1971) et 13 (1) (1972) nous indique une valeur de $t$ non significative. Les deux expériences sont comparables. Les valeurs obtenues sont semblables.

Nous avons également calculé les écarts-types et les coefficients de variation (Tableau 1). Le coefficient de variation de la teneur en acide céto-9 décène-2 oïque est élevé dans toutes les séries. Toutefois de la fin juin à la fin juillet ainsi qu'en hiver, de la fin novembre au début du mois de mars 1972, il est particulièrement important.

Les valeurs les plus faibles apparaissent au début de mai et au début de juin, période où l'on enregistre une augmentation des quantités d'acide céto-9 décène-2 oïque ainsi qu'en octobre et en novembre, période où l'on en obtient, au contraire, des quantités relativement faibles. Ces résultats montrent l'hétérogénéité du matériel biologique utilisé. Ils ne doivent pas surprendre lorsqu'on connaît l'importance de la variabilité génétique chez les abeilles et le rôle considérable des.facteurs du milieu.

\section{2. - Dosage de l'acide hydroxy-9 décène-2 oüque dans les têtes de reines âgées de $23 \pm 1$ jours}

Les quantités moyennes obtenues dans chaque série d'élevage sont portées sur la figure 1 (courbe B) et dans le tableau 1.

Pour les raisons déjà avancées précédemment, les résultats des deux dosages du mois de juin et ceux des deux dosages du mois de juillet ont été groupés dans la série 4 (séries $4+4^{\prime}$ de juin) et dans la séries 5 (séries $5+5^{\prime}$ de juillet).

Les variations quantitatives observées pour ces séries suivent celles de l'acide céto-9 décène-2 oïque : les quantités d'acide hydroxy-9 décène-2 oïque les plus fortes apparaissent ici encore dans les séries $4^{\prime}$ et $5^{\prime}$ (Tableau 1). La quantité la plus importante d'acide céto-9 décène- 2 oïque trouvée dans les têtes de reines de même âge se présente cependant un mois avant (courbe $A$, figure 1).

D'une façon générale, les quantités moyennes d'acide hydroxy-9 décène-2 oïque augmentent surtout à partir du mois de juin et jusqu'à la fin du mois de juillet. C'est durant cette période qu'on note la quantité la plus forte d'acide. Elle est cependant deux fois plus faible que la quantité d'acide céto-9 décène-2 oïque enregistrée à la même période.

Les quantités d'acide hydroxy-9 décène-2 ö̈que diminuent ensuite nettement au mois d'août où elles atteignent les valeurs les plus basses. Dès le début d'octobre, elles augmentent faiblement et sont plus importantes pendant les mois d'hiver (décembre à février) tandis que les quantités d'acide 
céto-9 décène-2 oïque les plus fortes se situent en décembre et janvier et qu'elles diminuent déjà en février.

Dans leur ensemble, les quantités d'acide hydroxy-9 décène-2 oïque obtenues sont beaucoup plus faibles que celles d'acide céto-9 décène-2 oïque. On savait déjà que dans la fraction acide méthylée d'un extrait de têtes de reines, l'acide hydroxy -9 décène- 2 oïque apparaît dans la proportion de $80 \mu \mathrm{g}$. pour $200 \mu \mathrm{g}$. d'acide céto-9 décène-2 oïque (Callow, Chapman, Paton, 1964).

Bien que les variations quantitatives observées soient de faible amplitude les valeurs obtenues laissent supposer l'existence d'un cycle avec un premier maximum en juillet et un second qui s'étale de décembre à février. Les valeurs sont toutefois légèrement décalées par rapport à celles du cycle de production de l'acide céto-9 décène 2 oïque. Nous discuterons plus loin de cette observation.

Dans l'ensemble, les coefficients de variation sont très élevés dans toutes les séries (Tableau 1). Nous notons qu'ils sont plus importants lorsqu'il s'agit des dosages de l'acide hydroxy-9 décène-2 oïque que lorsqu'il s'agit de ceux de l'acide céto-9 décène-2 oïque. Nous pensons que cela peut être dû au fait que l'acide hydroxy-9 décène-2 oïque apparaît chez les reines suivant certaines modalités de formation qui sont encore difficiles à expliquer.

Nous avons également effectué le test $\mathrm{t}$ de différence de moyennes entre les différents points mensuels de la courbe $B$ de la Fig. 1 (Tableau 5). Les résultats indiquent des valeurs significatives entre les séries 1 et 2,2 et 3 , 3 et 4 . Comme pour l'acide céto-9 décène 2 oïque, les quantités d'acide hydroxy9 décène-2 oïque augmentent aussi de mars à juin. De juin à juillet, les quantités d'acides restent les mêmes (test $t$ non significatif). Cependant lorsque l'on compare la série 2 à la série 5 , les valeurs sont très significatives $(P=1 \%$ oo $)$. On peut affirmer par conséquent que les quantités d'acide hydroxy-9 décène-2 oïque augmentent régulièrement du mois de mars au mois de juillet. Les quantités d'acide hydroxy-9 décène-2 oïque diminuent ensuite très significativement jusqu'à la fin d'août. De la fin d'août au début d'octobre, on note une augmentation très significative (comparaison des séries 6 et 7). Nous n'observons pas ce phénomène à propos du cycle de l'acide céto-9 décène-2 oïque. Nous obtenons ensuite des valeurs non significatives entre les séries 7 et 8 , 8 et 9,9 et 10,10 et 11,11 et 12. On pourrait alors supposer que les teneurs en acide hydroxy-9 décène-2 oïque n'augmentent pas en hiver. Toutefois lorsque l'on compare les séries 6 et 10,7 et 10,6 et 12, 7 et 12, les valeurs de $t$ sont significatives. On admet donc comme pour l'acide céto-9 décène-2 oïque, qu'il y a en hiver une augmentation des quantités d'acide hydroxy-9 décène-2 oïque. Elles ne sont pas d'ailleurs plus élevées que celles obtenues en juillet (test $t$ non significatif entre les séries 5 et 10, 5 et 12). Enfin, la comparaison entre les 2 séries 1 (1971) et 13 (1) (1972) nous donne une valeur significative 
$(\mathrm{P}=1 \%)$. Les deux expériences ne sont donc pas comparables pour cet acide. Ces résultats nous laissent supposer qu'il y aurait deux périodes (mars et octobre) où les ouvrières produisent une certaine quantité d'acide hydroxy-9 décène-2 oïque dont les modalités de sécrétion ne suivent pas tout à fait le rythme de production de l'acide céto-9 décène-2 oïque.

3. - Comparaison des quantités moyennes d'acides céto-9 et hydroxy-9 décène-2 ö̈que dans les têtes de reines issues de deux colonies éleveuses au cours d'une même série d'élevage

Au début de ce travail, nous avons indiqué (méthodes d'élevage de reines) que nous n'avons conservé en vue des dosages des deux acides phéromonaux que le groupe de reines issu du meilleur élevage. Au mois de février 1971, ne sachant pas si nous pourrions obtenir des reines, nous avons effectué simultanément deux élevages. De plus, à cette période de l'année, il était possible que les reines obtenues ne contiennent que des quantités d'acides difficilement dosables. C'est pourquoi, nous avons procédé aux dosages de deux groupes de reines dans la série 1 . Ils constituent les séries 1 et 1 bis.

Le tableau 2 indique le nombre de reines naissantes des 2 groupes et le pourcentage de réussite.

Dans le tableau 3, nous remarquons que les quantités moyennes d'acide céto- 9 décène- 2 oüque trouvées dans les têtes de reines des 2 séries 1 et 1 bis sont du même ordre de grandeur. Par contre, les quantités moyennes d'acide hydroxy-9 décène- 2 oïque ne le sont pas.

Nous nous sommes donc demandé dans quelle mesure, au cours d'une même série d'élevage, les valeurs obtenues sont susceptibles de varier en fonction de la ruche éleveuse. Nous avons donc procédé à une analyse de la variance.

Pour l'acide céto-9 comme pour l'acide hydroxy-9 décène- 2 oïque, nous obtenons une valeur de F. non significative. Nous constatons done que pour les deux colonies utilisées, les dosages d'acide céto-9 décène- 2 oïque chez les reines de $23 \pm 1$ jours donnent des résultats semblables et qu'il en est de même en ce qui concerne les dosages d'acide hydroxy-9 décène-2 oïque.

Par la suite, nous avons décidé de comparer dans la dernière série (série 13) les quantités d'acides phéromonaux des reines issues de plusieurs ruches éleveuses.

4. - Comparaison des quantités moyennes d'acides céto-9 et hydroxy-9 décène-2 öqque dans les têtes de reines issues de quatre colonies éleveuses, au cours de la dernière série d'élevage

Dans la dernière série de la fin de mars 1972, nous avons gardé les reines en provenance des quatre ruches éleveuses choisies pour la durée totale de 
TавL. 2. - Nombre de reines issues des deux groupes de la sërie 1 et des 4 groupes de la série 13. TAB. 2. - Zahl der aus d. beiden Gruppen d. Serie 1 und d. 4 Gruppen d. Serien 1-13 (1) hervorgegangenen 9 우

\begin{tabular}{|c|c|c|c|c|c|}
\hline $\begin{array}{l}\text { Numéro } \\
\text { de la colonie } \\
\text { éleveuse } \\
\\
\text { d. Pr. } \\
\text { Negevolks }\end{array}$ & $\begin{array}{c}\text { Date d'éclosion } \\
\text { des reines } \\
\text { Schlüpfdatum } \\
\text { d. 우 }\end{array}$ & $\begin{array}{c}\text { Nombre } \\
\text { de reines } \\
\text { naissantes } \\
\text { Zahl d. } \\
\text { geschlïpften 우 }\end{array}$ & \begin{tabular}{|c|} 
Nombre de reines \\
naissantes \\
par rapport au \\
nombre de larves \\
mises en élevage \% \\
Anzahl geschl. $9 \%$ im \\
Verhältnis zu d. \\
angebotenen Larven \\
$\%$
\end{tabular} & $\begin{array}{c}\text { Nombre } \\
\text { de reines } \\
\text { utilisées } \\
\\
\text { Zahl d. } \\
\text { verwendeten } q 9\end{array}$ & $\begin{array}{l}\text { Age des reines } \\
\text { (en jours) } \\
\text { Alter d. } q q \\
\text { in Tagen }\end{array}$ \\
\hline 1 & le 08-03-1971 & 20 & 86,9 & 14 & 22 à 23 \\
\hline $1 \mathrm{bis}$ & le 08-03-1971 & 14 & 60,9 & 8 & 22 à 23 \\
\hline $13(1)$ & du 27 au $30-03-71$ & 16 & 69,6 & 16 & 25 à 28 \\
\hline $13(2)$ & du 27 au $31-03-71$ & 21 & 91,3 & 21 & 26 à 28 \\
\hline $13(3)$ & du 27 au $28-03-71$ & 15 & 65,2 & 15 & 27 à 28 \\
\hline $13(4)$ & du 27 au $30-03-71$ & 18 & 78,3 & 18 & 28 à 31 \\
\hline \multicolumn{2}{|c|}{$\begin{array}{l}\text { Nombre total de reines } \ldots \ldots \ldots \\
\text { Gesamtzabl d. }\end{array}$} & 104 & & 92 & \\
\hline
\end{tabular}

l'expérience (séries 13 (1) (2) (3) (4)). Nous avons donc pu comparer les quantités moyennes des deux acides phéromonaux produites par les reines des quatre groupes ainsi constitués. Cette expérience a été conduite afin de vérifier que les teneurs en acides trouvées dans les têtes des reines élevées dans des colonies différentes restent malgré tout semblables.

Dans le tableau 3 sont portées les moyennes des quantités d'acides trouvées dans les têtes des reines de la série 13 (1) à (4).

Nous indiquons dans le tableau 2, le nombre de reines naissantes des quatre groupes et le pourcentage de réussite. Dans cette treizième série, les naissances se sont échelonnées sur plusieurs jours. Nous rappelons que les quatre ruches éleveuses choisies pour la production des reines ont élevé régulièrement pendant un an. Elles ont passé l'hiver dans le rucher couvert. Il n'est donc pas étonnant d'observer dans cette dernière série un étalement des éclosions, en rapport avec un certain épuisement des ouvrières éleveuses. Les dosages des sécrétions royales n'ont pu être effectués que dans les têtes de reines âgées de 27 à $28 \pm 2$ jours.

Nous avons noté sur les courbes $\mathbf{A}$ et $\mathbf{B}$ de la figure 1, les résultats de la série 13 (1). Cette colonie n'a pas élevé de reines depuis près de deux mois. Elle a été choisie en fonction de notre protocole expérimental. Pour la période 


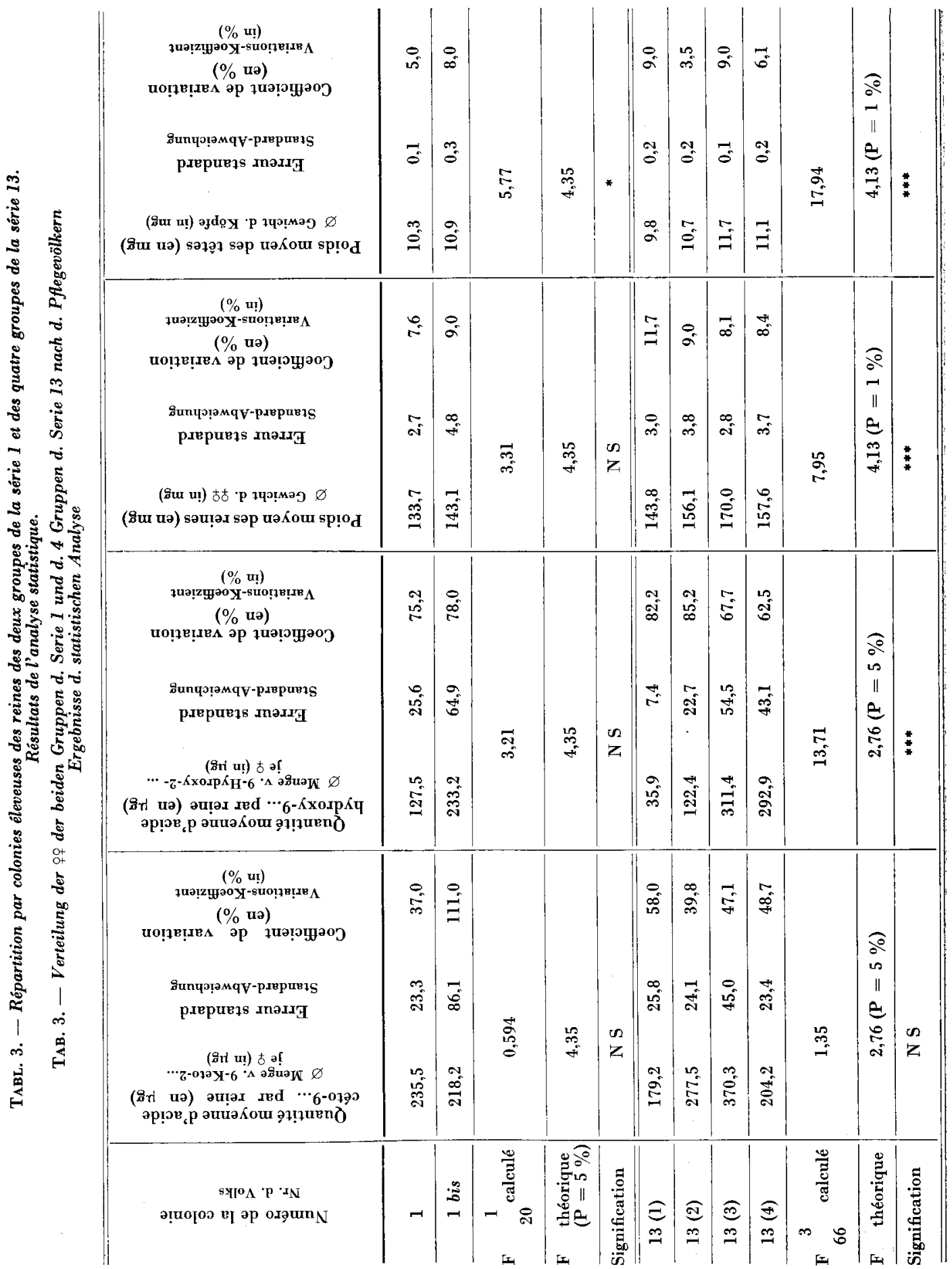


considérée, les colonies 13 (1) et 13 (3) ont élevé respectivement 16 et 15 reines. Nous avons dosé les reines du plus grand groupe.

Les quantités moyennes d'acide céto-9 décène- 2 oïque varient environ $d u$ simple au double. Les quantités d'acide hydroxy-9 décène-2 oïque sont encore plus variables puisqu'on note une valeur très basse dans la série 13 (1) et deux valeurs fortes dans les séries 13 (3) et (4) (Tableau 3).

Pour les deux acides, les coefficients de variation sont très élevés dans les quatre groupes de reines. Ils sont du même ordre de grandeur que ceux notés dans les différentes séries (Tableau 1).

Pour l'acide hydroxy-9 décène-2 oïque, le coefficient de variation est le plus élevé.

Pour l'interprétation des résultats, nous avons comme précédemment effectué une analyse de la variance (Test F.). Nous avons ensuite comparé les moyennes entre elles par le test $t$.

La valeur calculée de $\mathrm{F}$ n'est pas significative en ce qui concerne les quantités d'acide céto-9 décène- 2 oïque dosées par tête de reine dans chacune des colonies (Tableau 3). La comparaison des moyennes par le test t nous donne les mêmes résultats dans les différents groupes de reines comparés deux à deux. On peut donc considérer que les moyennes obtenues ont la même valeur. Les quatre colonies ont élevé des reines qui contiennent des quantités semblables d'acide céto-9 décène-2 oïque, pour la période d'élevage considérée.

Pour l'acide hydroxy-9 décène-2 oïque, la valeur calculée de F est hautement significative (Tableau 3). La comparaison des moyennes par le test $t$ nous indique qu'il n'y a pas de différences significatives entre les 2 séries 13 (3) et (4) et 13 (1) et (2). Par contre, la comparaison des moyennes entre les séries $13(1)$ et (4), $13(2)$ et (4), $13(1)$ et (3), $13(2)$ et (3) indique des valeurs significatives $(\mathrm{P}=1 \%$ ). Les moyennes obtenues n'ont donc pas la même valeur. Les quatre colonies ont élevé des reines dont la teneur en acide hydioxy9 décène- 2 oïque est très variable.

On constate donc une bonne stabilité des résultats au cours des dosages de l'acide céto- 9 décène- 2 oïque dans les têtes des reines âgées de $23 \pm 1$ jours bien qu'elles proviennent de colonies différentes. Ces résultats sont d'ailleurs identiques à ceux de la série 1 et 1 bis. Il n'en est pas de même en ce qui concerne les dosages de l'acide hydroxy-9 décène 2-oïque.

Les naissances s'étant échelonnées sur plusieurs jours, nous avons réparti par classes d'âge les reines issues des quatre colonies. Nous n'avons pas tenu compte des dosages effectués chez les quelques reines nées en début et en fin d'éclosion.

Dans le tableau 4, les données de la série 13 (1) à (4) sont classées sèlon l'âge des reines et non plus selon la ruche d'origine. 
On constate alors que les quantités d'acide céto-9 décène-2 oïque sont analogues et que, par contre, pour l'acide hydroxy-9 décène-2 oïque, il y a des différences sensibles. Les coefficients de variation sont élevés et varient selon l'âge des reines. Ils sont les plus élevés lorsque l'on considère les quantités moyennes d'acide hydroxy-9 décène-2 oïque.

Nous avons effectué de la même façon une analyse de la variance (test $F$ ) puis la comparaison des moyennes obtenues, par âge, en utilisant le test t.

Pour l'acide céto-9 décène-2 oïque, la valeur calculée de F n'est pas significative pour les reines âgées de 26 à 29 jours. La comparaison des moyennes. par le test $t$ nous donne les mêmes résultats dans les différentes classes d'âge comparées 2 à 2 . On peut envisager que les moyennes obtenues sont comparables.

La production d'acide céto-9 décène-2 oïque ne semble donc pas en rapport avec l'âge des reines, tout au moins pour l'écart d'âge considéré ici qui est de 4 jours, et pour des reines âgées de plus de 26 jours.

Pour l'acide hydroxy-9 décène-2 oïque, la valeur calculée de $F$ n'est pas significative. La comparaison des moyennes par le test $t$ donne des résultats identiques. Cependant après transformation des données quantitatives en $100 \log _{10}^{x}$, la valeur de $F$ devient significative au seuil de $5 \%\left(F_{60}^{3}\right.$ $=2,87>2,76)$. Il existe done de petites différences entre les reines en fonction de l'âge quant à la production d'acide hydroxy-9 décène-2 oïque.

\section{5. — Étude des variations de poids chez les reines vierges maintenues en captivité}

a) Variations des poids frais des reines entières.

Les poids frais les plus bas sont observés en début de saison, dans la série 1 (mars 1971) et la série 13 (1) (mars 1972). Ils atteignent respectivement $133,7 \mathrm{mg}$ et $143,8 \mathrm{mg}$. Ils augmentent ensuite jusqu'au mois de mai. Le poids maximum est, en mai, de $180,9 \mathrm{mg}$ (série 3). A partir du mois de juin, on observe une diminution progressive des poids jusqu'à la fin novembre (série 8 et 9). Dans le tableau 1 bis, on note cependant dans la série no 8 (mi-novembre) une légère augmentation des poids. Elle pourrait être en rapport avec le nombre très faible de reines obtenues dans cette série. Les reines mieux nourries pèseraient plus lourd. Sur la courbe $A$, figure 2 , nous constatons ensuite qu'en décembre (série 10), il y a une très faible remontée des poids suivie d'une diminution jusqu'en mars 1972.

D'après le tableau 1 bis, les valeurs du coefficient de variation sont importantes au mois de mars et au mois d'avril. Elles sont les plus faibles en mai, juin et début juillet. Les valeurs sont à nouveau importantes de la fin 
MISE EN ÉVIDENCE D'UN CYCLE SAISONNIER

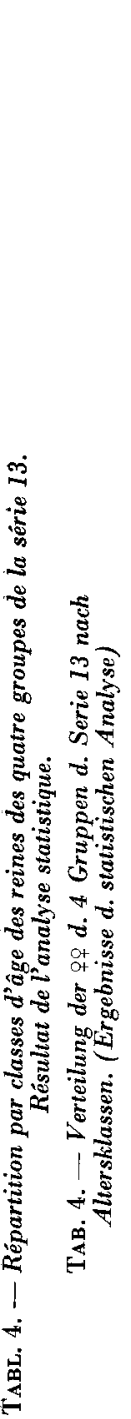

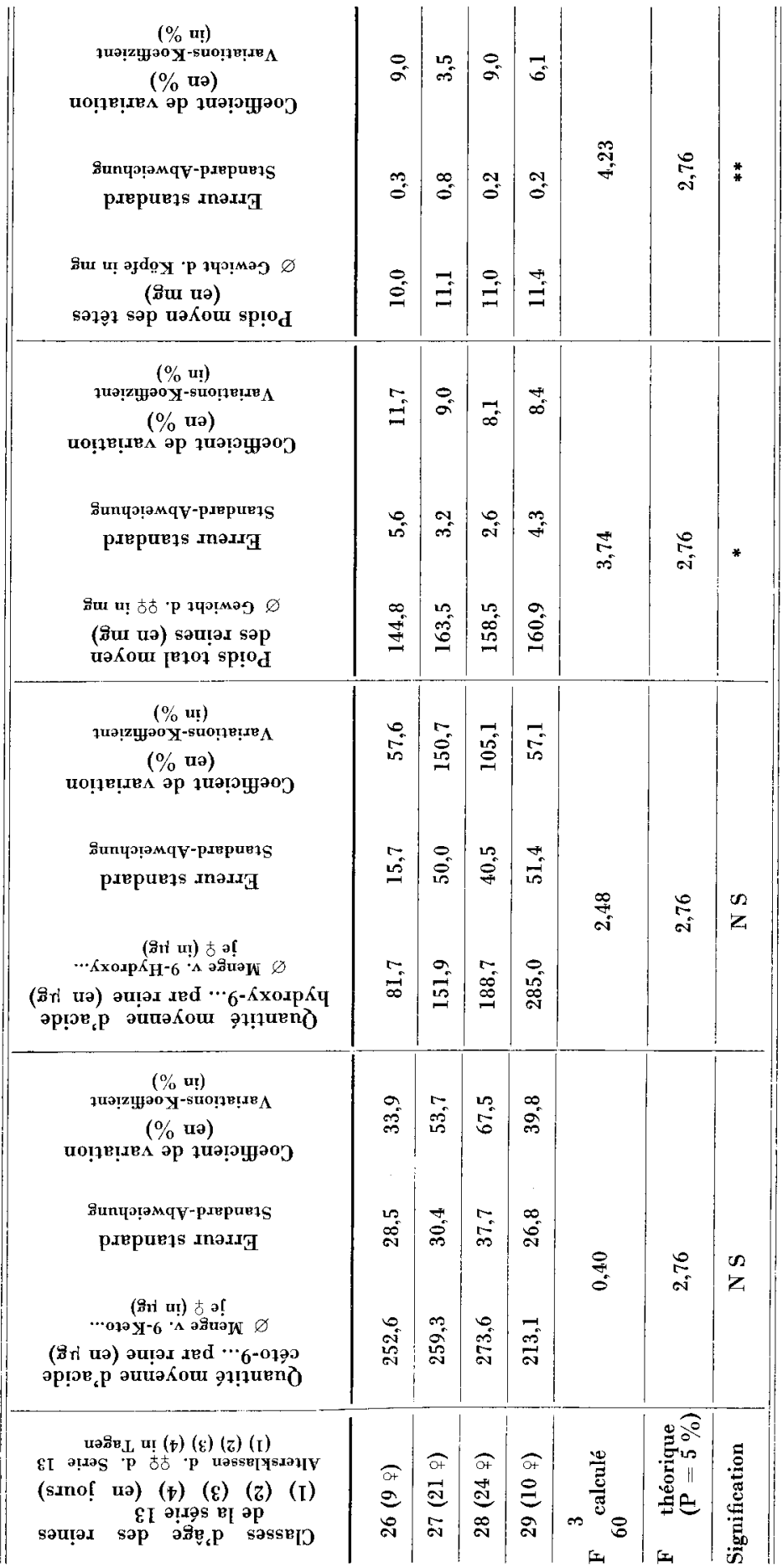


juillet à la fin de novembre et de la fin janvier au mois de mars 1972. La valeur des écarts-types suit la même évolution.

Chaque reine ayant fait l'objet d'une pesée individuelle, nous avons pu calculer pour chaque série la variance et effectuer un test $t$ de différence de moyennes entre les différents poids moyens mensuels de la courbe A (figure 2, Tableau 5). Les résultats indiquent des valeurs significatives entre les séries 1 et 2, 2 et 3 . On peut donc parler d'une augmentation des poids du mois de mars au mois de mai. Les poids diminuent ensuite à partir du mois de juin et jusqu'à la fin de novembre. Les valeurs non significatives indiquent seulement que les poids évoluent peu au cours de cette période. Toutefois, si nous effectuons un test $t$ entre les séries 3 et 5,3 et 6,3 et 7,3 et $8,+9$ réunies constituant la série de novembre, nous constatons que les valeurs calculées sont très significatives. Par conséquent, les poids diminuent régulièrement jusqu'en novembre. La série de novembre (série 8 et 9) comparée à la série de décembre (série 10) donne une valeur de t non significative. Les poids sont d'ailleurs peu différents (moyennes de 156,2 $\mathrm{mg}$ pour les séries 8 et 9 de novembre et de $162,4 \mathrm{mg}$ pour la série 10). A partir de décembre, les poids diminuent jusqu'en mars (test $\mathrm{t}$ très significatif $(\mathrm{P}=1 \% \mathrm{oo})$ ) entre les séries 10 et 13 (1). Nous avons ensuite comparé les poids obtenus entre la première et la dernière série mais nous n'obtenons pas des valeurs comparables (t significatif à $\mathrm{P}=5 \%$ ). Nous avions obtenu pour ces mêmes reines des quantités d'acide céto-9 décène- 2 oïque tout à fait comparables entre les 2 séries 1 et 13 (1). La courbe des poids des reines entières suit done une évolution qui ne paraît pas se calquer exactement sur les courbes de production des acides. Pour les poids, le maximum apparaît un mois avant celui de l'acide céto-9 décène-2 oïque et deux mois avant celui de l'acide hydroxy-9 décène 2 oïque. D'autre part, il n'y a pas en hiver, une augmentation bien nette des poids moyens. Les séries extrêmes ne se recoupent pas. L'augmentation des poids moyens des reines pendant la belle saison doit suivre le processus général du développement des colonies mais il n'y a pas de rapport bien net entre le poids des reines d'une part et leur contenu en acides phéromonaux d'autre part.

b) Variations des poids frais de la tête des reines.

Les poids de la tête sont portés sur la figure 2. Pour chaque série, ils correspondent à la différence du poids moyen des reines (courbe A) et du poids moyen du thorax et de l'abdomen (courbe B).

D'après l'écart qui existe entre les deux courbes, nous constatons une évolution générale du poids des têtes qui paraît être la même que celle du poids des reines entières. Les poids maxima se situent en mai et juin et atteignent $11,8 \mathrm{mg}$. (Tableau 1 bis, séries 3 et $4+4^{\prime}$.)

Les valeurs du coefficient de variation sont importantes $\mathrm{du}$ mois de mars 
au mois de mai. Elles sont les plus faibles en juin et juillet. Les valeurs sont à nouveau importantes de la fin septembre à la fin décembre 1971 et de la fin janvier au mois de mars 1972 (Tableau 1 bis). Dans leur ensemble (sauf pour la série 10), les valeurs du coefficient de variation sont plus faibles que celles des poids des reines entières. La valeur des écarts types suit la même évolution. Les valeurs les plus élevées se placent sensiblement aux mêmes périodes que celles des poids des reines entières.

On admet que les variations pondérales sont localisées dans le thorax et l'abdomen. Hoopingarner et Farrar (1959) trouvèrent que les variations du poids des reines sont dues à des variations de poids de l'abdomen. La courbe $B$ (fig. 2) représentant l'évolution du poids du thorax et de l'abdomen est semblable à la courbe $\mathrm{A}$ (fig. 2) des poids des reines entières, ce qui confirme les données des auteurs précédents.

c) Comparaison des poids des reines issues de quatre colonies éleveuses, au cours de la dernière série d'élevage.

Nous avons comparé les poids des corps entiers et des têtes des reines vierges issues des quatre ruches éleveuses de la série 13 (1) (2) (3) (4). Nous désirions contrôler les variations pondérales de reines en provenance de colonies différentes.

Le tableau 3 indique les poids moyens des reines entières et ceux des têtes. Les pesées ont été effectuées chez des reines âgées de 27 à $28+2$ jours, conservées à l'état frais dans un congélateur. Les dates d'éclosion des reines et le nombre de reines obtenues dans les quatre groupes d'élevage sont indiqués dans le tableau 2.

Les coefficients de variation sont élevés et différents entre eux. La variation est certainement en rapport avec l'échelonnement des éclosions. Nous signalons que les pesées n'ont pas été effectuées dans le but d'étudier en particulier les variations de poids des reines.

Le coefficient de variation est le plus faible dans les têtes des reines.

Nous avons effectué une analyse de la variance (test $F$ ) suivi d'un test $t$. L'analyse des données porte sur le poids moyen des reines de chacune des. colonies ainsi que sur le poids moyen des têtes (en $\mathrm{mg}$ ).

La valeur calculée de $F$ est hautement significative $(1 \%$ aussi bien pour les poids des reines entières que pour les poids des têtes. La comparaison des moyennes par le test $t$ indique des valeurs significatives entre les séries 13 (1) et $(2), 13(1)$ et (3), $13(1)$ et (4), $13(2)$ et (3), 13 (3) et (4). Les deux moyennes de la série 13 (2) et (4) ne sont pas significatives. Elles correspondent d'ailleurs aux deux groupes de reines où les poids ont des valeurs presque semblables.

Les quatre colonies éleveuses ont donc produit des reines dont les poids 
sont très variables d'une ruche à l'autre. Ce résultat est certainement en rapport avec le fait que les reines utilisées en vue des pesées présentent un écart d'âge, sans doute trop important, de 2 à 4 jours. Nous pensons que les reines perdent encore du poids lorsqu'elles sont claustrées même après le $25^{\mathrm{e}}$ jour. On suppose que le poids des reines diminue régulièrement au cours de leur séjour en étuve et que cette perte est en relation avec le vieillissement des ouvrières accompagnatrices.

Dans le tableau 4, les poids sont répartis par classe d'âge. Les différences pondérales sont ainsi légèrement réduites. Les coefficients de variation restent cependant élevés. Ils sont à peu près du même ordre de grandeur que ceux évalués dans chacune des colonies.

La valeur de $\mathbf{F}$ est significative entre les reines âgées de 26 à 29 jours. La comparaison des moyennes par le test $t$ donne des valeurs significatives entre les groupes d'âges de 26 et 27,26 et 28,26 et 29 jours. Les moyennes non significatives apparaissent entre les groupes de reines âgées de 27 et 28, 27 et 29 , 28 et 29 jours. On constate donc que la moyenne des poids des reines âgées de 26 jours se détache significativement des autres moyennes. Il se peut que cela soit dû au nombre peu élevé de reines âgées de 26 jours (Tableau 4). Les poids moyens des reines et des têtes n'ont donc pas la même valeur selon que les reines sont âgées de $26,27,28$ ou 29 jours.

\section{6. - Études des variations des pourcentages d'acceptation de larves}

Sur la figure 3 , nous avons noté les pourcentages de larves royales acceptées ainsi que les pourcentages de reines naissantes obtenues dans la colonie nous ayant donné le plus grand nombre de reines. Ce sont ces reines qui ont été conservées en vue des dosages. Les données des séries de juin (4i et $4^{\prime}$ ) et de juillet ( 5 et $5^{\prime}$ ) sont portées séparément sur Jes courbes $\mathbf{A}$ et $\mathbf{B}$ de la figure 3 . Le pourcentage de reines naissantes suit de très près le pourcentage de larves acceptées.

Pendant les mois d'été, nous observons deux périodes d'élevage : une première période qui apparaît du mois de mars au début de juillet avec un maximum de larves acceptées au début de juin $(100 \%)$, une deuxième période qui se place en septembre.

Pendant les mois d'hiver, dans les conditions du rucher couvert, nous obtenons une troisième période d'élevage. Elle apparaît en décembre et se poursuit jusqu'au printemps.

Nous notons deux chutes dans les pourcentages de larves royales acceptées : à la fin de juillet et à la mi-novembre. Au cours de cette dernière période, 
nous avons eu beaucoup de difficultés à prélever et choisir les jeunes larves. Nous avons cependant toujours transféré le même nombre de 23 larves.

Nous pensons que l'aspect des courbes reflète bien les conditions naturelles d'élevage de reines de la région parisienne, pour la période normale de reproduction.

Si nous comparons la courbe des poids moyens des reines entières (fig. 2, courbe A) à la courbe des pourcentages d'acceptation de larves (fig. 3, courbe A), il semble qu'il existe un certain rapport entre les deux courbes. Le maximum de larves acceptées se place au début du mois de juin, le minimum à la minovembre. Si l'on tient compte du décalage dans le temps entre le poids des reines noté au 23 e jour et le nombre de larves royales acceptées, nous considérons que les augmentations et les diminutions observées sur les courbes se placent sensiblement aux mêmes moments de l'année.

\section{DISCUSSION}

Il est intéressant en premier lieu de comparer nos résultats avec ceux? des autres auteurs qui ont quantifié la production des acides phéromonaux chez les reines et qui ont effectué des mesures de poids.

Les quantités d'acide céto-9 décène- 2 oïque que nous avons trouvées dans les têtes de reines vierges âgées de 23 jours sont nettement supérieures aux quantités indiquées par les autres auteurs. Les différences constatées sont dues au fait que nous avons utilisé des reines vierges dont on a contrôlé l'origine et l'âge alors que les chercheurs étrangers ont prélevé des reines de provenances diverses, fécondes ou vierges, d'âge variable provenant dans la plupart des cas de colonies normales. Les reines que nous avons examinées sont gardées en cagette expérimentale en présence de petites populations. Elles n'ont donc pas été au contact d'un grand nombre d'ouvrières et la demande en substances phéromonales n'est certainement pas la même. Sa distribution ou son stockage dans la glande mandibulaire a pu influencer les quantités dosées, dans les têtes de reines.

Les quantités d'acide hydroxy-9 décène- 2 oïque sont inférieures à celles d'acide céto-9 décène-2 öqque. Elles apparaissent dans le même rapport que celui indiqué par les auteurs anglais (Callow, Chapman, Paton, 1964). Nous remarquons que l'écart entre les deux courbes est le plus réduit dans la période allant de février à avril (fig. 1 , courbes $A$ et B). Il est le plus grand en juin. Nous supposons que ces oscillations seraient en relation avec la biosynthèse cyclique de l'acide céto-9 décène- 2 oïque.

Le poids des reines vierges encagées pendant 23 jours est plus faible que celui des reines pesées à l'éclosion (Mirza, Duduman, Dragan, 1967). Les 
reines vierges encagées perdraient donc du poids en étuve. Bien que les conditions de captivité des reines ne soient pas exactement les mêmes, KHarcheva (1957) trouva des résultats semblables aux nôtres. SAHoval et Krieg (1971) effectuant des pesées de reines âgées de 1 à 3 jours ont montré qu'après l'éclosion les poids diminuent rapidement.

Les variations quantitatives observées au cours d'une année, nous ont permis de mettre en évidence l'existence de cycles saisonniers des acides et des poids.

1) Le cycle de l'acide céto-9 décène-2 oïque est à notre avis, le plus remarquable et le plus stable. Nous avons noté un premier maximum de la sécrétion au mois de juin. La présence de plus de $1000 \mu \mathrm{g}$. dans les têtes de reines de la série 4' de la fin de juin nous laisse supposer que la phéromone est loin d'agir à très petites doses comme le ferait une hormone.

Un premier minimum se situe de la fin d'août jusqu'au début d'octobre; un second apparaît au mois de mars.

Un deuxième maximum est atteint au cours du mois de décembre. Les reines nées au cours de cette période d'élevage inhabituelle contiennent cependant deux fois plus d'acide qu'il n'est signalé dans la littérature.

2) Le cycle de l'acide hydroxy-9 décène-2 oïque est moins accentué et paraît moins stable que celui de l'acide céto-9 décène- 2 oüque. Le maximum de la sécrétion se situe en juillet. L'observation d'un décalage dans le temps entre les maxima de sécrétion de l'acide céto-9 décène-2 oïque et de l'acide hydroxy-9 décène- 2 oïque, de même qu'un étalement de la courbe obtenue pour ce dernier (courbe B, fig. 1) pourrait être en faveur d'une hypothèse précédemment émise par Johnston, Law, Weaver (1965) à savoir que l'acide céto-9 décène-2 oïque transmis aux ouvrières, serait transformé par celles-ci en plusieurs acides dont l'acide hydroxy-9 décène-2 oüque. Ce dernier serait ensuite redonné aux reines. L'existence de ce cycle d'oxydo-réduction pourrait peut-être expliquer le décalage observé. Il n'exclut pas la possibilité d'avoir également chez la reine un processus d'oxydo-réduction identique : les reines transformeraient elles-mêmes une partie de l'acide céto-9 décène-2 oïque en d'autres acides comme l'acide hydroxy-9 décène-2 oïque. Elles pourraient peut-être élaborer aussi l'acide céto- 9 décène- 2 oïque à partir de l'acide hydroxy- 9 décène- 2 oïque produit dans les glandes mandibulaires.

Il existe un cycle des poids. Le maximum se situe en mai avant que n'apparaissent les quantités les plus fortes des deux acides phéromonaux. Selemenev (1970) le place en juin. Sкrobal (1958) indique une diminution vers l'automne. Shimanova (1966) a noté qu'en fonction des particularités de l'année (importance de la miellée, apports de pollen, conditions météorologiques favorables), on peut obtenir des reines naissantes dont le poids le plus 
important est au début de la saison ou en fin de saison. L'évolution des poids paraît être surtout un problème de nutrition. Au cours de nos expériences, à partir de la naissance, les reines ont été placées dans les mêmes conditions d'élevage en cagette. Les facteurs nutritionnels ont dû par conséquent agir avant la naissance. Il est possible que les larves destinées à devenir des reines n'aient pas été nourries de façon homogène. Des différences qualitatives et quantitatives dans la nourriture distribuée aux larves ainsi que des variations dans le nombre des nourrices en rapport avec les facteurs extérieurs pourraient expliquer en partie la grande variabilité des poids et les coefficients élevés de variation.

Après avoir mis en évidence l'existence de cycles saisonniers des acides et des poids, nous nous sommes demandé comment des variations de cet ordre avaient pu apparaître chez des reines claustrées dont on avait contrôlé les conditions de captivité.

Bien que les reines aient été placées dès leur naissance dans une étuve où elles ont toujours reçu la même nourriture, en présence du même nombre d'ouvrières maintenues à une température constante, elles ont subi l'influence des facteurs extérieurs au cours de leur élevage larvaire et au cours de leur vie d'adulte.

Au stade larvaire les reines dépendent des nourrices qui se trouvent dans les colonies du rucher couvert. Ces ouvrières, bien que maintenues dans un rucher protégé, peuvent sortir et de ce fait, réagir aux variations de température et de luminosité, et récolter une nourriture plus ou moins abondante. Les larves de reines sont donc alimentées plus ou moins bien selon la saison.

Déjà à ce stade, elles reçoivent les substances nécessaires à la formation ultérieure des acides phéromonaux et en particulier l'acide hydroxy-10 décène-2 oïque contenu dans la gelée royale et considéré comme le précurseur de l'acide céto-9 décène-2 oïque (Brown, Fela uER, 1961).

A l'état adulte, les reines vierges sont mises en présence de deux sortes d'ouvrières. Les premières, peu nombreuses, d'âge variable, proviennent directement de la colonie éleveuse de reines. Ces abeilles sont nées et sont restées un certain temps dans les colonies du rucher couvert. Elles ont donc participé aux échanges de nourriture, à l'élevage du couvain et à différents travaux en relation avec les facteurs extérieurs. Ce sont ces ouvrières qui vont s'occuper des reines au moment de leur naissance. Les secondes plus nombreuses, âgées de quelques jours au début de l'expérience, proviennent de cadres de couvain naissant à l'étuve. Or ce couvain a été élevé à différentes périodes de l'année. Les ouvrières naissantes accompagnatrices des reines ne sont pas dans le même état physiologique selon qu'elles sont issues de cadres de couvain naissant en mai ou en janvier. De ce fait, elles peuvent modifier le rythme de production des substances phéromonales émises par les reines. 
Ainsi, bien que les reines aient toujours été placées dans les mêmes conditions depuis leur naissance, elles sécrètent les substances phéromonales suivant un rythme saisonnier que leur imposent les ouvrières. L'in fluence du milieu n'a donc pas été totalement éliminée au cours de nos expériences.

Notre attention a été attirée par le fait qu'il existe un parallélisme étroit entre le cycle de production de l'acide céto-9 décène-2 oïque par les reines (Pain, Roger, Theurkauff, 1972) et celui de l'acide hydroxy-10 décène-2 oïque par les ouvrières (PaIn, Roger, 1970) (courbe C, fig. 1).

Pour les deux types d'expériences, la préparation du matériel vivant n'a pas été la même. Nous constatons cependant que les deux acides présentent le même cycle annuel.

Ces résultats ne paraissent pas surprenant étant donné les liens biochimiques qui existent entre ces substances (Brown, FELAUER, 1961). Ils confirment que ce sont les ouvrières qui ont été introduites avec les reines qui ont joué un rôle prépondérant dans le cycle de production des acides phéromonaux. Il apparaît de plus en plus nettement que le rôle de l'ouvrière est primordial. Nous pensons qu'il ne se limite pas seulement à régir la production des substances actives des reines mais doit également influencer les poids et les pourcentages d'acceptation de larves royales. L'étude de l'évolution de tous ces processus au cours d'une année est très probante lorsqu'on examine de plus près les différentes périodes mensuelles :

Ainsi d'avril à mai et juin, période où les élevages de reines et les poids sont en augmentation, les quantités d'acides céto-9, hydroxy-9 et hydroxy-10 décène- 2 oïque croissent pour atteindre un maximum qui se place en juin pour les acides céto-9 et hydroxy-10 décène- 2 oïque.

Nous supposons qu'à ce moment-là, les substances phéromonales bien que produites en très grandes quantités par la reine ne comblent pas les besoins de la population car cette période correspond à l'expansion des colonies. C'est en effet, l'époque où l'on enregistre une remontée des températures, un accroissement de la luminosité, un regain de la végétation. Nous sommes en période de jours croissants. Ces phénomènes se répercutent sur le poids des ruches, les quantités d'acides et la production de couvain (fig. 4, courbes A et B). Les surfaces de couvain les plus grandes apparaissent en juin (Louveaux, 1966). La population très importante est composée surtout de jeunes ouvrières. Leur renouvellement est rapide car ce sont des abeilles d'été à durée de vie courte. De nombreuses ouvrières nourrices apparaissent. Elles apportent à la reine des substances dont l'acide hydroxy-10 décène-2 oïque. La reine doit avoir atteint son maximum de production d'acides phéromonaux. C'est l'époque des essaims où l'on note l'apparition de nombreuses cellules royales.

En juillet, août et septembre, le pourcentage d'acceptation de larves royales bien qu'encore très important (75 à $90 \%$ ) n'atteint toutefois pas les valeurs du 
mois de juin $(100 \%)$. Le poids des reines diminue ainsi que les quantités d'acides royaux et d'acide hydroxy-10 décène-2 oïque. Les quantités les plus faibles de ces 3 acides apparaissent en août et septembre. Cette période correspond au déclin des colonies. A partir de juillet, nous sommes en période de baisse de la température et de la luminosité (fig. 4). On assiste à une diminution du poids des ruches. La production de couvain est ralentie. La population est par conséquent moins forte. Les ouvrières sont encore de jeunes abeilles mais elles se préparent déjà à l'hivernage. Leur renouvellement est moins rapide qu'en été. On peut les considérer comme des ouvrières dont la physiologie est intermédiaire entre celle des abeilles d'été et celle des abeilles d'hiver typique à durée de vie longue. Ces ouvrières sont pauvres en acide hydroxy-10 décène-2 oïque. I'aspect des courbes (fig. 1) indique que des teneurs très faibles en cet acide entraîne chez les reines des teneurs basses en acide céto- 9 décène- 2 oïque.

$A$ partir d'octobre, nous assistons à une baisse très nette du nombre de cellules royales acceptées et du nombre de reines naissantes. Le poids des reines continue à diminuer. Les quantités des deux acides phéromonaux sont peu importantes. A la même période, la production d'acide hydroxy-10 décène-2 oïque est la plus faible. Les colonies continuent à perdre du poids. La quantité de couvain est réduite. La population est constituée d'ouvrières nées en septembre et octobre qui ont été nourries par des ouvrières nées en juillet et août, à une période où nous avons enregistré (PAIN, RogER, 1970) une chute brutale

FIG. 4

(A) évolution saisonnière :

de la luminosité (inspirée de Danilevskir (1965)

de la température (inspirée de Louveatix (1966)

de la végétation (représentation schématique)

du poids des ruches (inspiré de Louveaux (1966)

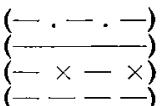

(B) êvolution saisonnière

du couvain (inspiré de Louveaux (1966))

de la population (représentation schématique)

de l'acide céto-9 décène- 2 oüque

de l'acide hydroxy-10 décène 2 oïque

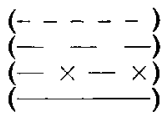

Авв. 4

(A) = Jahreszeitliche Entwicklung :

d. Helligkeit (nach DANILEvSKII 1965)

d. Temperatur (nach Louveaux 1966)

d. Vegetation (schemat. Darstellung)

d. Gewichts d. Stöcke (n. Louveaux 1966)

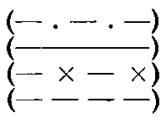

(B) = Jahreszeitliche Entwicklung :

d. Brut (nach Louveaux 1966)

des Volkes (schemat. Darstellung)

d. 9-Keto-2-decensäure

d. 10-Hydroxy-2-decensäure

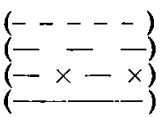



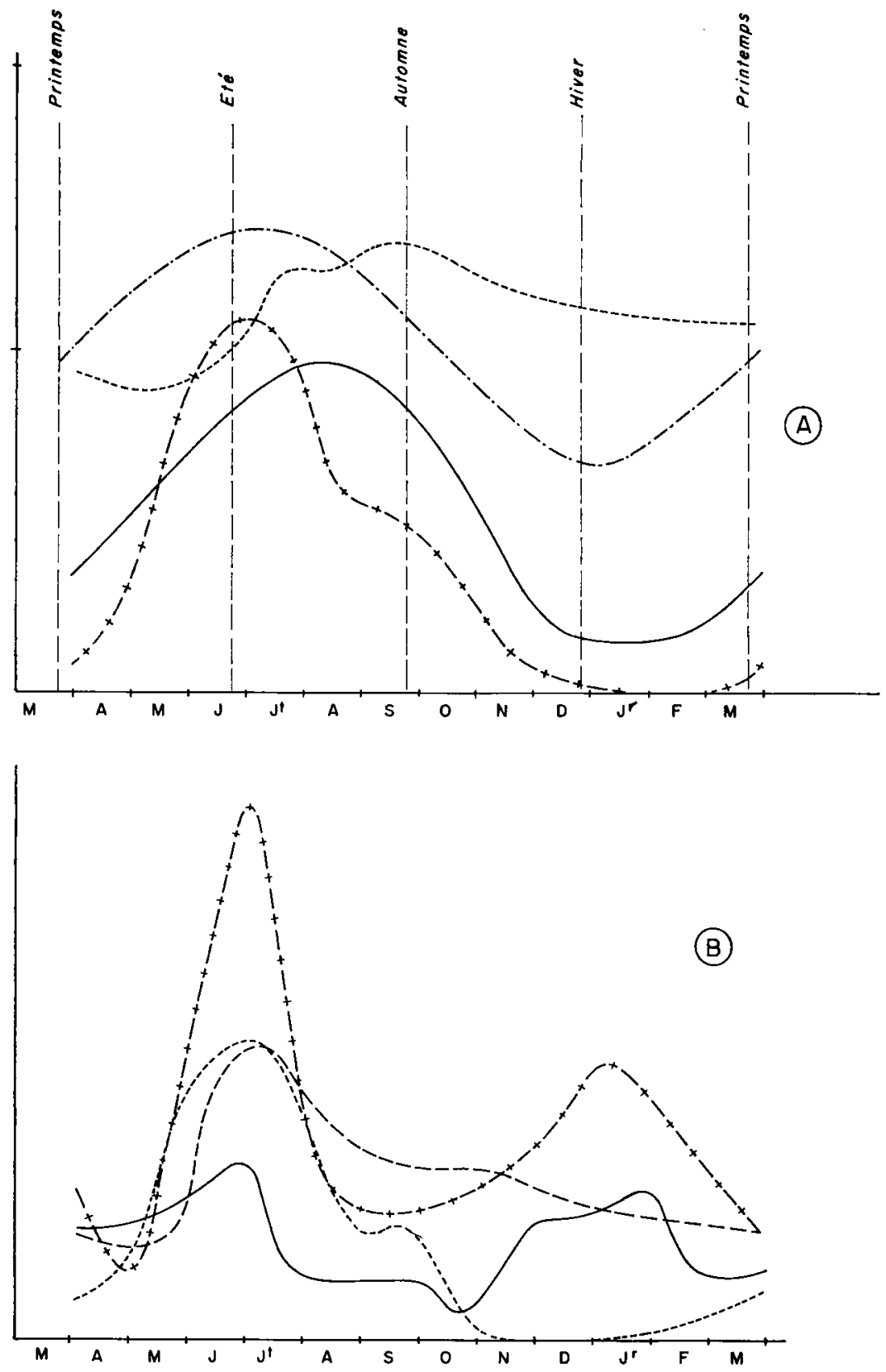
des valeurs en acide hydroxy-10 décène-2 oïque. Les ouvrières accompagnatrices des reines sont donc des nourrices pauvres en acide hydroxy-10 décène-2 oïque. Elles ne sont pas en état de distribuer aux reines la ou les substances indispensables à la formation des acides phéromonaux.

Vers la fin de novembre et en décembre, nous constatons une deuxième période favorable aux élevages de reines. Les pourcentages de réussite en décembre sont aussi importants qu'en août et septembre (fig. 3). Le poids des reines augmente très légèrement (fig. 2). Par la suite, il diminue significativement jusqu'en mars. Il y a done, au cours de cette période, une augmentation parallèle des élevages et du poids des reines. En même temps, nous enregistrons un deuxième maximum de la production des deux acides phéromonaux avec un étalement de l'acide hydroxy-9 décène-2 oïque (fig. 1 , courbe $B$ ). La quantité d'acide hydroxy-10 décène-2 oïque est en forte augmentation chez les ouvrières (fig. I, courbe C).

L'importance des teneurs en phéromones nous paraît être en rapport avec le maintien de la grappe hivernale. C'est la période où les colonies sont les moins populeuses. Il n'y a pas ou presque pas de renouvellement des ouvrières. La plupart sont des abeilles âgées car la production de couvain est nulle (fig. 4, courbe B). Nous avons montré (Roger, PaIN, 1968 - Pain, Roger, 1970) qu'en hiver, l'absence de couvain et de renouvellement des ouvrières entraîne une augmentation des teneurs en acide hydroxy-10 décène-2 oïque. Les ouvrières accompagnatrices se trouvent dans une phase d'accumulation de cet acide, qui correspond à une augmentation des teneurs en acides phéromonaux (fig. 1, courbes A et B). Il est possible que la demande en substances phéromonales soit peu élevée voir nulle au cours de cette période. Les phéromones s'accumuleraient dans les glandes mandibulaires des reines. Nous avions indiqué qu'en hiver, les reines fécondes sont très attractives (PAIN, 1961). Les ouvrières moins actives, car cette période correspond à une baisse générale de la température et de la luminosité, (période de jours décroissants) s'occupent peu de la reine et la lèchent moins (Allen, 1957 - Skirkjavichudus, 1968). La reine conserverait donc ses phéromones. Les résultats des dosages confirment ces hypothèses du stockage des substances phéromonales.

Dans le courant de janvier et de février, les élevages de reines sont un peu moins bons, les pourcentages de réussite plus faibles par rapport à ceux de décembre. Les reines continuent à perdre du poids. La quantité d'acide céto-9 décène-2 oïque décroît, celle d'acide hydroxy-9 décène-2 oïque reste stationnaire pour les raisons indiquées auparavant. Les quantités d'acide hydroxy-10 décène-2 oïque dans les têtes des ouvrières âgées de 16 jours sont faibles.

Nous sommes à nouveau en période de jours croissants. La température s'élève et la luminosité est plus forte (fig. 4, courbe A). Les colonies commencent à évoluer car la reine accentue sa ponte. La population encore composée d'ou- 
vrières âgées est moins apte à élever des reines. Les jeunes abeilles sont encore en nombre insuffisant pour les élever correctement. Les reines ne reçoivent pas en quantité suffisante les substances indispensables à la formation des acides dont elles vont avoir besoin à partir de mars et d'avril.

Nos résultats nous permettent de supposer que les cycles que nous avons mis en évidence chez les reines encagées d'Apis mellifica ligustica S. sont représentatifs d'un processus général que l'on devrait pouvoir retrouver chez les reines fécondes de colonies. Seules, des variations d'ordre quantitatif seraient susceptibles de modifier les valeurs obtenues.

D'une manière générale, nous pouvons conclure que c'est en juin que les processus étudiés sont au maximum de leur évolution. Il en est de même pour d'autres processus physiologiques d'origine glandulaire. Différents auteurs ont en effet constaté pendant la période d'été l'existence d'un maximum de sécrétion dans les glandes mandibulaires des ouvrières pour l'heptanone, la bioptérine et l'acide pantothénique (Hanser, Rembold, 1964 - Boch, Shearer, 1967).

Nous soulignerons pour terminer que les dosages que nous avons effectués depuis plusieurs années contribuent dans une large mesure à nous faire mieux comprendre le cycle biologique annuel des colonies d'abeilles en rapport avec la biochimie des sécrétions phéromonales.

$$
\begin{gathered}
\text { Reçu pour publication en juin } 1974 . \\
\text { Eingegamgen im Juni } 1974 .
\end{gathered}
$$

\section{REMERCIEMENTS}

Les auteurs remercient M. J. Mesquida de la Chaire de Zoologie de l'École Nationale Supérieure Agronomique de Rennes qui a bien voulu effectuer une partie du travail de Statistique ainsi que Mme J. Proust du Laboratoire de Biologie Expérimentale (Université de Paris, Faculté des Sciences d'Orsay) qui nous a donné des conseils au sujet de ces analyses.

\section{ZUSAMMENFASSUNG}

Nachdem ein jahreszeitlicher Zyklus des Gehalts an 10-Hydroxy-2-decensäure in den Köpfen der Bienenarbeiterinnen nachgewiesen wurde, haben wir uns die Aufgabe gestellt, die Mengen der Königinnenpheromone (9-Hydroxy-2-decensäure und 9-Keto-2-decensäure), die in den Köpfen unbegatteter Königinnen von Apis mellifica ligustica S. vorhanden sind, zu bestimmen. Da diese drei Säuren biochemische Verwandte sind, stellten wir die Hypothese auf, dass, wenn eine von ihnen (10-Hydroxy-2-decensäure) einen jahreszeitlichen Rhythmus aufweist, es sich mit den beiden Königinnenpheromonen ebenso verhalten könne.

Ausserdem haben wir, da die Untersuchung der jahreszeitlichen Gewichtsveränderungen der Königinnen bisher nur Gegenstand einiger weniger Arbeiten war, Königinnen und Köni- 
ginnenköpfe gewogen und zwar zu dem Zeitpunkt, an dem sie für die Pheromonbestimmungen vorbereitet wurden.

Die Technik der gas-chromatographischen Bestimmung von 9-Keto-2-decensäure und 9-Hydroxy-2-decensäure ist die gleiche, wie sie schon für die 10-Hydroxy-2-decensäure beschrieben wurde (PAIN, Roger, 1970). Die Köpfe der Königinnen werden in Äther zerrieben; nach dem Filtrieren werden die Extrakte konzentriert, danach methyliert. Zu den getrockneten Estern gibt man entsprechende Mengen Hexan.

Die Bestimmungen der Kopfextrakte erhält man durch Vergleich der Lage der aufgezeichneten Piks mit der der Proben von Methylestern der synthetischen 9-Keto-2-decensäure und 9-Hydroxy-2-decensäure. Die erhaltenen Werte sind in $\mu \mathrm{g}$ je Biene angegeben, die Gewichte in $\mathrm{mg}$ berechnet.

Die jungen Larven, die zur Königinnenzucht gebraucht wurden, sowie die schlüpfenden Begleitbienen entstammen Völkern, die in einem Bienenhaus der Anstalt gehalten werden. Die Bienenstöcke werden von Oktober bis April bei einer konstanten Temperatur von $20^{\circ} \mathrm{C}$ gehalten. Während dieser Zeit erhalten die Völker aus dem laufenden Jahr stammende Pollenhöschen, die in frischem Zustand bei $-25^{\circ} \mathrm{C}$ aufbewahrt werden.

Während eines Jahres haben wir fortlaufend Serien von Königinnen mit einmaligem Umlarven gezogen. Die sehr jungen, noch undifferenzierten Larven entstammen in den meisten Fällen dem gleichen Italienervolk. Sie werden in einem weisellosen Volk der gleichen Rasse gep flegt. Die Entweiselung ist den äusseren Entwicklungsbedingungen der Völker angepasst.

1971 haben wir insgesamt vier Pflegevölker mit einjährigen Geschwisterköniginnen benutzt. Bei jeder Zuchtserie erhalten die Völker je zwei Gruppen von 23 Larven. Mit Rücksicht auf die durchzuführenden Bestimmungen behalten wir nur die Königinnen des Volkes mit der grössten Anzahl angenommener Larven. Im Verlauf der folgenden Serie sichern die beiden anderen Pflegevölker dann die Zuchten und so fort, bis Ende März 1972. Vor dem Schlüpfen werden die Königinnen einzeln in Versuchskäfige gebracht (PAIN, 1966), in denen sich 15 aus dem Pflegevolk stammende Arbeiterinnen unbestimmten Alters befinden. Nach dem Schlüpfen der Königinnen gibt man vierzig 1 bis 4 Tage alte Arbeiterinnen dazu. Die Königinnen bleiben bei $32 \pm 1{ }^{\circ} \mathrm{C}$ im Brutschrank, bis sie $23 \pm 1$ Tag alt sind. Dann werden sie abgetötet und einzeln in Röhrchen bei $-25{ }^{\circ} \mathrm{C}$ aufbewahrt bis zum Zeitpunkt des Wägens und der Bestimmungen. Die Königinnen wurden Einzelbestimmungen unterzogen, was uns erlaubte, die Standardabweichung und den Variationskoeffizienten zu berechnen. Die Kurven geben die mittleren Werte jeder Zuchtserie und das Geburtsdatum der Königinnen an.

Wir erhielten regelmässig jeden Monat junge Königinnen, zuweilen zweimal in einem Monat (Serien 4 u. 5, Serien 8 u. 9). Weiterhin haben wir im Verlauf der beiden Serien alle geschlüpften Königinnen aufbewahrt, sowohl die der beiden Pflegevölker (Serie 1) als auch die der vier Pflegevölker (Serie 13), um die Pheromonmengen und die Königinnengewichte der verschiedenen Zuchten vergleichen zu können.

Die Untersuchung von 199 jungen Königinnen in 13 Serien beweist das Vorhandensein eines jahreszeitlichen Zyklus des Pheromongehaltes und des Gewichts der Königinnen.

1. Der Zyklus von 9-Keto-2-decensäure ist am deutlichsten ausgeprägt und am stabilsten. Er scheint unabhängig vom Pflegevolk zu sein. Die Untersuchung der Ergänzungsserien (Serie l f und Serie 13 (2) (3) (4) zeigt, dass die Säuremengen statistisch gleich sind, wenigstens für die Untersuchungszeit (März 1971 und März 1972). Weiterhin decken sich die beiden Märzserien. Ein erstes Sekretionsmaximum tritt im Juni auf, ein zweites, weniger bedeutendes, wird im Dezember erreicht. Die geringsten Mengen treten im August/September und im März auf.

2. Der Zyklus der 9-Hydroxy-2-decensäure deckt sich teilweise mit dem von 9-Keto-2decensäure, weist allerdings einige Besonderheiten auf. Die erhaltenen Werte sind deutlich 
geringer und sind im Verhältnis zu denen der produzierten 9-Keto-2-decensäure leicht verschoben. Sie zeigen eine gewisse Instabilität; die Säuremengen sind in der Serie 13 untereinander statistisch verschieden, je nachdem, ob die Königinnen aus Volk 1, 2, 3 oder 4 stammen. Ausserdem decken sich die beiden Märzserien nicht. Der Variationskoeffizient ist bedeutender als der von 9-Keto-2-decensäure. Die beobachteten Unterschiede lassen sich erklären, wenn man die von Johnston, LaW und Weaver (1965) aufgestellte Hypothese in betracht zieht. Ein erstes Sekretionsmaximum tritt im Juli auf, ein zweites, gleich bedeutendes zeigt sich von Dezember bis Februar. Die geringsten Mengen wurden im August registriert.

3. Der Zyklus der Königinnengewichte scheint nicht in vollem Einklang mit dem der Pheromonerzeugung. Das Maximum tritt im Mai auf, also einen Monat vor dem der 9-Keto-2decensäure und acht Wochen vor dem der 9-Hydroxy-2-decensäure. Die Gewichte nehmen dann regelmässig bis zum November ab. Im Winter gibt es, anders als bei den Säuren, keine bemerkenswerte Zunahme bei den Gewichten. Sie nehmen bis zum März ab, aber die Königinnengewichte vom März 1971 und 1972 weisen bedeutende Unterschiede auf. Weiterhin sind die Gewichte von Stock zu Stock sehr verschieden und weisen Unterschiede je nach dem Alter der Königinnen auf (Serie 13).

Der Zyklus der Kopfgewichte gleicht in der Entwicklung dem der Königinnengewichte. Der Wert des Variationskoeffizienten ist sehr gering.

Es scheint sine gewisse Beziehung zwischen der Entwicklung der Königinnengewichte und dem Prozentsatz der angenommenen Larven zu bestehen (Abb. 2 u.3).

Die beschriebenen quantitativen Veränderungen bei unbegatteten, 23 Tage gekäfigten Königinnen scheinen von den Arbeiterinnen auszugehen, die selbst dem Ein fluss äusserer Faktoren unterliegen.

Tatsächlich hängen die Arbeiterinnen im Larvenzustand von den Pflegebienen ab, die auf jahreszeitlich bedingte Veränderungen reagieren. Als Adulte sind die Königinnen von den mit ihnen gekäfigten, aber zu einem anderen Zeitpunkt als sie selbst geschlüpften Begleitbienen abhängig. Ihr physiologischer Zustand ändert sich der Jahreszeit entsprechend, dadurch können sie den Rhythmus der Pheromonerzeugung verändern. Die Königinnen sezernieren somit die Pheromonsubstanzen nach einem jahreszeitlichen Rhythmus, der ihnen von den Arbeiterinnen aufgezwungen wird.

Es besteht ein enger Parallelismus zwischen dem Zyklus der Erzeugung von 10-Hydroxy-2decensäure durch die in einem Stock gehaltenen Arbeiterinnen (PAIN, Roger 1970) und dem der Produktion von 9-Keto-2-decensäure durch die unbegatteten Königinnen, die in Käfigen im Thermostat gehalten werden (Pain, Roger, Theurkauff 1972). Die Sekretion der beiden Säuren folgt dem gleichen Jahreszyklus. Die den Königinnen beigegebenen Arbeiterinnen spielen demnach eine entscheidende Rolle im Zyklus der Pheromonbildung.

In der gemässigten Zone weisen die Zyklen der Säuren und der Gewichte unbestreitbar Bindungen an den biologischen Jahreszyklus der Bienenvölker auf.

Von April bis Mai/Juni wachsen die Mengen der 9-Hydroxy-2-decensäure und der 10Hydroxy-2-decensäure an. Die Aufzucht der Königinnen und die Gewichte nehmen zu. Wir räumen ein, dass zu diesem Zeitpunkt die in grossen Mengen produzierten Königinnenpheromone den Bedürfnissen des Volkes, das an Stärke zunimmt und hauptsächlich aus jungen Sommerbienen besteht, nicht entsprechen. Diese liefern der Königin Substanzen, von denen eine 10-Hydroxy-2-decensäure ist. Die Temperatur steigt an, die Helligkeit nimmt zu. Es ist die Zeit der Schwärme (Kurven, Abb. 4).

Im August und September sind die drei Säuren nur in geringen Mengen vorhanden. Die Königinnengewichte nehmen ab. Die Volksdichte ist weniger stark. Die Arbeiterinnen bereiten sich auf die Überwinterung vor. Sie sind arm an 10-Hydroxy-2-decensäure. Im Oktober spitzt sich dieser Zustand zu. 
Gegen Ende November und im Dezember ist eine parallel verlaufende Zunahme der Bruttätigkeit, der Königinnengewichte und der drei Säuren zu verzeichnen. Wir vermuten, dass die Nachfrage nach Pheromonen gering ist, weil die Königin, die wenig beleckt wird, ihre Pheromone behält. Das zahlenmässig schwächere Volk setzt sich aus alten Arbeiterinnen zusammen, die sich in einer Phase der Anhäufung von 10-Hydroxy-2-decensäure befinden (Pain, Roger, 1970). Die Temperatur ist abgesunken und die Helligkeit vermindert. Es ist die Zeit, in der die Arbeiterinnen die Wintertraube bilden.

Die jahreszeitlich bedingte Erzeugung der untersuchten Säuren dürfte deutlich genug zu Tage getreten sein, um jetzt bei der Italienerbiene vom Vorhandensein eines Jahreszyklus der Pheromone bei unbegatteten Königinnen sprechen zu können.

\section{RÉFÉRENCES BIBLIOGRAPHIQUES}

AlLen M.-D., 1957, Observations of honeybees examining and licking their queen. Brit. J. Animal Behav., 5, 81-84.

Barbier M. et al., 1960, Auftrennung der sauren Anteile von Extrakten aus Bienenköniginnen (Apis mellifica L.), Isolierung des als Königinnen-Substanz bezeichneten Pheromones. Helv. chim. acta, XLIII, 6, 1682-1689.

Barbier M., Pain J., 1960, Étude de la sécrétion des glandes mandibulaires des reines et des ouvrières d'abeilles (Apis mellifica) par chromatographie en phase gazeuse C.R. Acad. Sci., $250,3740-3742$.

Barbier M., HÜGEL M.-F., 1961, Synthèses dans la série de l'acide céto-9 décène-2 trans-oïque (substance royale). Bull. Soc. Chim. Fr., 951-954.

Boch R., Shearer D.-A., 1967, 2 Heptanone and 10 Hydroxy-Trans-Dec. 2 Enoï Acid in the Mandibular glands of worker Honey Bees of different ages. Z. vergleich. Physiol., 54,1-11.

Butler C.-G., Callow R.-K., Johnston N.-C., 1961, The isolation and synthesis of queen substance, 9 oxodec-trans-2-enoic acid, a honeybee pheromone. Proc. r. Soc., Ser. B, 155, $417-432$.

Butler C.-G., Paton N.-P., 1962, Inhibition of queen rearing by queen honeybees (Apis mellifera L.) of different ages. Proc. r. entomol. Soc. London, ser. A, 37, 7-9.

Butler C.-G., Fairey E.-M., 1964, Pheromones of the honeybee : biological studies of the mandibular gland secretion of the queen. J. apic. Res., 3, 2, 65-76.

Butrer C.-G., Callow R.-K., Chapman J.-R., 1964, 9 hydroxydec-trans-2-enoic acid, a Pheromone stabilizing honey bee swarms. Nature, London, 201 (4920), 733.

Butler C.-G., 1967, Insect Pheromones. Biol. Rev., 42, 42-87.

Butler C.-G., Calam D.-H., Callow R.K., 1967, Attraction of Apis mellifera drones by the odours of the queens of two other species of honeybees. Nature, London, 213, (5074), 423-4.24.

Butler C.-G., Callow R.K., 1968, Pheromones of the honeybee (Apis mellifera L.). The " inhibitory scent " of the queen. Proc. r. entomol. Soc. London, ser. B, 43 (4-6) 62-65.

Brown W.-H., Felauer E.-E., 1961, A new fatty acid from royal jelly. Nature, London, 190 (4.770) 88.

Brown W.H., Felauer E.E., Smith M.-V., 1962, Biosynthesis of royal jelly acid from sucrose. Nature, London, 195 (4836), 75-76.

Callow R.-K., Johnston N.-C., 1960, The chemical constitution and synthesis of queen substance of honeybees. (A.M.). Bee World, 41, 152-153.

Callow R.-K., Chapman J.R., Paton P.-N., 1964, Pheromones of the honeybee : chemical studies of the mandibular gland secretion of the queen. J. Apic. Res., 3, (2), 77-89.

Danilevskir A.-S., 1965, Photoperiodism and Seasonal Development of Insects. translated by Johnston J. and Waloff N. Oliver and Boyd. Edinburgh and London, p. 33.

Eiter K., 1962, Neue Synthesen der Königinnen Substanz und der 9-hydroxy 2 trans decensaüre-(1). Liebigs Ann. Chem., 658, 91-99.

GARY N.-E., 1962, Chemical mating attractants in the queen honeybee. Science, 36, 773-774. 
Hanser G., Rembold H., 1964, Analytische und histologische Untersuchungen der Kopf und Thoraxdrüsen bei der Honigbiene Apis mellifica. Z. Naturforsch., B, 19 (10), 938943.

Hoopingarner R., Farrar C.-L., 1959, Genetic control of size in queen honey-bees. J. econ. Entomol., 52 (4), 547-548.

Johnston N.-C., Law J.-H., Weaver N., 1965, Metabolism of 9-Ketodec-2-enoïc Acid by Worker Honeybees. Biochemistry, 4, 1615-1621.

Kharcheva A.-I., 1957, Change in the weight of queens kept in cages with workers. Bee World, 4, 59, 104 .

LouveauX J., 1966, Les modalités de l'adaptation des abeilles (Apis mellifica L.) au milieu naturel. Ann. Abeille, 9, 4, 323-350.

Mirza F., Duduman S., Dragan M., 1967, Variation saisonnière du poids des reines à l'éclosion. (en roumain). Apicultura, Bucuresti, 20, (8), 2-6.

Pain J., 1961, Sur la Phérormone des reines d'abeilles et ses effets physiologiques. Paris, Institut National de la Recherche Agronomique, 70 p. (Thèse Sc. Nat., Paris 1961, no 4.526).

PAin J., 1966, Nouveau modèle de cagettes expérimentales pour le maintien d'abeilles en captivité. Ann. Abeille, 9 (1), 71-76.

Pain J., Barbier M., Roger B., 1967, Dosages individuels des acides céto-9 décène-2 oïque et hydroxy-10 décène-2 oïque dans les têtes des reines et des ouvrières d'abeilles. Ann. Abeille, 10 (1), 45-52.

Pain J., Roger B., 1970, Variation annuelle de l'acide hydroxy-10 décène-2- oïque dans les têtes d'abeilles. Apidologie, 1, (1), 29-54.

Pain J., Roger B., Theurkauff J., 1972, Sur l'existence d'un cycle annuel de la production de phéromone (acide céto-9 décène-2 oüque) chez les reines d'abeilles (Apis mellifica ligustica Spinola). C.R. Acad. Sci., D, 275, 2399-2402.

PaIN J., 1973, Pheromones and hymenoptera. Bee World, 54 (1), 11-24.

Roger B., 1971, L'influence de la reine d'abeille (Apis mellifica L.) sur la prise de nourriture des ouvrières accompagnatrices. Apidologie, 2, (2), 123-155.

Roger B., Pain J., 1968, Le couvain, facteur déterminant de la teneur en acide hydroxy-10 décène-2 oïque de la tête des ouvrières d'abeilles (Apis mellifica L.) C.R. Acad. Sci., D, Paris, 266, 2267-2269.

Sahoval J., Krieg P., 1971, Le changement du poids des reines d'abeilles après l'éclosion. Apimondia, 23e Congrès Int. Apic. Moscou, 434.

Sannasi A., Sundara rajulu G., 1971, 9 oxo-trans-2-decenoïc acid in he Indian honeybees. Life Sci., 10 (4) part. 2, 195-201.

Selemenev S.-I., 1970, Influence de la période de sortie des reines des abeilles de l'ExtrêmeOrient sur leurs caractères extrêmes et sur le volume des cellules mères. (en russe). Itogi elssper. Rabot molod. Issledovatel. Vopr. sel'shs. Khoz, 18, 160-163.

ShEARER D.-A., et al., 1970, Occurence of 9-oxodec-trans-2-enoï acid in queens of Apis dorsata, Apis cerana and Apis mellifera. J. Insect Physiol., 16 (7), 1437-1442.

Shimanova I.-P., 1966, Variation saisonnière du poids des reines vierges à l'éclosion des abeilles de montagnes du Caucase et de Russie centrale dans la région de Ryazan. (en russe). Uchen. Zap. ryazansk gos. pedagog Inst., 47, 27-31.

SkirkJavichuus A.-V., 1968, Comportement des ouvrières d'abeilles autour de leur reine. (en russe) Trudy Akad. Nauk Litor S.S.R., ser. B, 2 (46), 85-95.

Skrobar D., 1958, Poids des reines, des mâles et des ouvrières d'abeilles. (en tchèque). Ved. Prace Vyzkum. Ustav. Vcelar. C. S.A. ZV., I, 151-164.

Vuillaume M., 1958, Techniques d'élevages de reines. I - Le premier stade : élevage des cellules royales. Étude critique. Ann. Abeille, 3, 189-196.

WEIss K., 1967, Zur vergleichenden Gewichtsbestimmung von Bienenköniginnen. Z. Bienenforsch., $9(1), 1-21$. 